\title{
ZrBDC-Based Functional Adsorbents for Small-Scale Methane Storage Systems
}

\author{
Olga V. Solovtsova (D), Ilya E. Men'shchikov $\mathbb{D}^{D}$, Andrey V. Shkolin (D), Anatoly A. Fomkin $\mathbb{D}^{\text {, }}$ \\ Elena V. Khozina $(\mathbb{D}$, and Andrey A. Shiryaev 1
}

M.M. Dubinin Laboratory of Sorption Processes, A.N. Frumkin Institute of Physical Chemistry and Electrochemistry,
Russian Academy of Sciences, Moscow 119071, Russia

Correspondence should be addressed to Olga V. Solovtsova; o.solovtsova@phyche.ac.ru

Received 7 September 2021; Revised 7 December 2021; Accepted 14 December 2021; Published 30 January 2022

Academic Editor: Eloy S. Sanz P rez

Copyright (c) 2022 Olga V. Solovtsova et al. This is an open access article distributed under the Creative Commons Attribution License, which permits unrestricted use, distribution, and reproduction in any medium, provided the original work is properly cited.

\begin{abstract}
Metal-organic frameworks (MOF), potentially porous coordination structures, are envisioned for adsorption-based natural gas (ANG) storage, including mobile applications. The factors affecting the performance of the ANG system with a zirconiumbased MOF with benzene dicarboxylic acid as a linker (ZrBDC) as an adsorbent were considered: textural properties of the adsorbent and thermal effect arising upon adsorption. The high-density ZrBDC-based pellets were prepared by mechanical compaction of the as-synthesized MOF powder at different pressures from 30 to $240 \mathrm{MPa}$ at $298 \mathrm{~K}$ without a binder and mixed with polymer binders: polyvinyl alcohol (PVA) and carboxyl methylcellulose (CMC). The structural investigations revealed that the compaction of ZrBDC with PVA under $30 \mathrm{MPa}$ was optimal to produce the ZrBDC-PVA adsorbent with more than a twofold increase in the packing density and the lowest degradation of the porous structure. The specific total and deliverable volumetric methane storage capacities of the ZrBDC-based adsorbents were evaluated from the experimental data on methane adsorption measured up to $10 \mathrm{MPa}$ and within a temperature range from 253 to $333 \mathrm{~K}$. It was measured experimentally that at $253 \mathrm{~K}$, an $100 \mathrm{~mL}$ adsorption tank loaded with the ZrBDC-PVA pellets exhibited the deliverable methane storage capacity of $172 \mathrm{~m}^{3}(\mathrm{NTP}) / \mathrm{m}^{3}$ when the pressure dropped from 10 to $0.1 \mathrm{MPa}$. The methane adsorption data for the ZrBDC powder and $\mathrm{ZrBDC}-\mathrm{PVA}$ pellets were used to calculate the important thermodynamic characteristic of the $\mathrm{ZrBDC}_{\mathrm{CCH}} \mathrm{Cdsorption}$ system-the differential molar isosteric heat of adsorption, which was used to evaluate the state thermodynamic functions: entropy, enthalpy, and heat capacity. The initial heats of methane adsorption in powdered $\mathrm{ZrBDC}$ evaluated from the experimental adsorption isosteres were found to be $\sim 19.3 \mathrm{~kJ} / \mathrm{mol}$, and then these values in the $\mathrm{ZrBDC} \mathrm{CH}_{4}$ system decreased at different rates during adsorption. In contrast, the heat of methane adsorption onto the ZrBDC-PVA pellets increased from $19.4 \mathrm{~kJ} / \mathrm{mol}$ to a maximum with a magnitude, width, and position depended on temperature, and then it fell. The behaviors of the thermodynamic state functions of the $\mathrm{ZrBDC} / \mathrm{CH}_{4}$ adsorption system were interpreted as a variation in the state of adsorbed molecules determined by a ratio of $\mathrm{CH}_{4}-\mathrm{CH}_{4}$ and $\mathrm{CH}_{4}-\mathrm{ZrBDC}$ interactions. The heat of adsorption was used to calculate the temperature changes of the ANG systems loaded with ZrBDC powder and ZrBDC pellets during methane adsorption under adiabatic conditions; the maximum integrated heat of adsorption was found at $273 \mathrm{~K}$. The maximum temperature changes of the ANG system with the ZrBDC materials during the adsorption (charging) process did not exceed $14 \mathrm{~K}$ that are much lower than those reported for the systems loaded with activated carbons. The results obtained are of direct relevance for designing the adsorption-based methane storage systems for the automotive industry, developing new gas-power robotics systems and uncrewed aerial vehicles.
\end{abstract}

\section{Introduction}

A wealth of modern studies is devoted to finding solutions to reduce carbon dioxide emissions from power plants, which increase the amount of greenhouse gases in the atmosphere and contribute to global climate change [1]. One of the ways to solve this problem is to replace liquid hydrocarbon fuels, which are refined petroleum products, with natural gas 
(NG), which contains more than 70 percent methane and produces the lowest carbon dioxide emissions. There are proven reserves of 47,805 billion cubic meters of NG in Russia [2]. Therefore, there is considerable scope for expanding NG use, including energy systems of heating and electricity generating plants and onboard vehicular applications (motor vehicles and aircraft). However, at normal temperature and pressure (NTP), NG has a lower energy density than that of gasoline and diesel, and this is a major setback to bringing NG into widespread use as a fuel [3-5]. At the same time, in many respects, the available technologies of compressed $(\mathrm{CNG})$ and liquified natural gas (LNG) fail to meet efficiency and safety requirements [6-8]. Therefore, new technological solutions are required to increase the energy density of NG, and an adsorbed NG (ANG) method seems to be the most effective one for accumulation and storage [9-11]. Based on a rich set of experimental data reported over the past twenty years [11-18], activated carbons and metal-organic framework structures are recognized as the most promising adsorbents for employing in ANG systems.

For example, the textural properties of the most efficient activated carbons as MAXSORB-III [19] can provide the specific methane storage capacity of over $200 \mathrm{~m}^{3}(\mathrm{NTP}) / \mathrm{m}^{3}$ at the pressure of $3.4 \mathrm{MPa}$ and $293 \mathrm{~K}$. However, a further increase in the efficiency of carbon adsorbents is limited mainly by the standard methods of their synthesis, including carbonization and activation. It is known that an increase in the activation degree of a carbon material induces an increase both in the volume of micropores and the specific surface area [20]. However, it inevitably leads to the expansion of pores and reduces the packing density [21], which negatively affects its specific total methane capacity.

Meanwhile, the synthesis of metal-organic frameworks (MOF) proceeds according to a fundamentally different mechanism, and the final sorption behaviors depend mainly on the structural parameters of a porous cluster formed by an organic ligand-metal ion pair, which makes it possible to form a variety of MOF structures with various properties. Wilmer et al. [22] and later Simon et al. [23] performed the screening analysis of more than a hundred thousand hypothetical MOF compounds in order to establish the relationship between the structural parameters and adsorption properties for methane. They found an optimal MOF structure that ensures the DOE 2012 target value of total methane adsorption capacity for a monolith crystal of 263$266 \mathrm{~m}^{3}(\mathrm{STP}) \cdot \mathrm{m}^{-3}$ at $3.5 \mathrm{MPa}$ and $298 \mathrm{~K}$ [15].

Many studies of note reported on the methane adsorption in MOF, which attained or even went ahead of the DOE target achievement by changing the thermodynamic conditions: either by increasing pressure [24] or decreasing temperature $[25,26]$. Moreover, there is a discrepancy in the theoretical calculations and experimental data caused by incorrect use of the crystal density of as-prepared powdered MOF in calculating a specific volumetric methane adsorption capacity [27]. Indeed, most MOF compounds are synthesized as crystals ranging in size from tens to hundreds of microns, and the resultant sample is a polydisperse microcrystalline powder. However, the use of MOF powders as adsorbents does not allow achieving the high productivity of the ANG system due to their unsatisfactory performance: low values of packing density and mechanical resistance, insufficient rates of mass and heat transfer, adsorption cyclical instability, formation of dust. The low packing density does not provide the high adsorption capacity of an ANG tank (or adsorber) loaded with the MOF powder. Therefore, for the practical application of the MOF-based materials in the ANG systems, it is necessary to develop methods to increase their packing density via shaping [3, 28-30]. The advantages of the shaped MOF materials are that they display better operational features such as high packing density and mechanical strength, humidity resistance, thermal conductivity, and resistance to physical stress, compared to the initial powders [30-32].

One of the approaches to MOF shaping implies in situ growth of MOF films on a porous substrate via the layer by layer method [33], atomic layer deposition [34], or their combination [35]. Thakkar et al. [36] reported on the successful application of the 3D printing technology to fabricate the MOF-74(Ni) and UTSA-16(Co) monoliths with MOF contents of up to 80 and $85 \mathrm{wt} . \%$, respectively. Posttreatment strategies employing the mixing of presynthesized MOFs with additives followed by microwave-assisted polymerization [37] or freeze-drying [38, 39] are proposed to fabricate shaped MOF-based composites with different MOFloadings and densities. Tian with coworkers enabled to prepare a monolithic porous Cu-based MOF (HKUST-1) with an increased density by sol-gel process [40]. The monolithic MOF showed robust mechanical properties and increased methane uptake.

Another approach implies mechanical shaping of MOF powders: dry or wet granulation [41], spray-drying [42], and pressing or extrusion under high pressures [43]. Mechanical pressing (compaction) of MOF powder is the simplest, flexible, and affordable technique used to prepare shaped MOF materials with higher density, thermal conductivity, and improved resistance to abrasion [30]. Two options for preparing MOF-based pellets or granules are possible. First, a binder is used to fabricate MOF pellets, which facilitates the shaping process and improves the mechanical strength and wear resistance of MOF-based material $[44,45]$. Another approach is to treat an assynthesized MOF powder without any binders [46, 47]. However, the compaction of MOF powders, especially under high pressures, the granulation, or extrusion shaping can cause irreversible degradation of the porous structure of MOF, and as a result, deterioration of the sorption performance [46]. Nandasiri with coworkers reported [48] that the shaping of different MOFs such as MIL-101 (Cr), MIL53 (Al), MOF-5, and HKUST-1 reduced the specific surface area, $S_{\mathrm{BET}}$, by $50 \%$ and the volume of pores by $\sim 43 \%$.

However, zirconium-based MOFs, including that containing a 1,4-benzene dicarboxylic acid (BDC)-ZrBDC or UiO-66-type MOF $[49,50]$, show high resistance to chemical attack, thermal, and mechanical stresses [51]. The adsorption capacity of the ZrBDC crystalline powder depends significantly on the activation conditions [50]. $\mathrm{ZrBDC}$ decomposes in the air at $\sim 350^{\circ} \mathrm{C}$, while in a vacuum, 
the decomposition occurs at temperatures above $450^{\circ} \mathrm{C}$, and it is resistant to boiling water and can withstand low concentrations of $\mathrm{H}_{2} \mathrm{~S}, \mathrm{SO}_{\mathrm{x}}$, and $\mathrm{NO}_{\mathrm{x}}$ [52]. It was found that the shaping of the ZrBDC powder under $665 \mathrm{MPa}$ did not significantly affect the structure; its specific surface and micropore volume decreased by no more than $2 \%$ [53].

It is worthwhile noting that the major impediments to large-scale industrial use of MOFs are the complexity of the synthesis process, the high cost of reagents, and, accordingly, the final product [54]. In this regard, it is advisable to link the prospects of using MOFs with their application as an adsorbent to store alternative fuels in small-scale energy systems as uncrewed air vehicles [55, 56]. Optimal shaping of MOF powders into a tailorable form is a significant step towards the implementation of this engineering solution.

In the previous studies $[28,57,58]$, we tested the conditions for preparing high-density functional materials from Cu-based MOF (CuBTC, where BTC is 1,3,5-benzene tricarboxylic acid) and ZrBDC. The primary purpose of the studies was to develop an optimal technique for fabricating the shaped materials from CuBTC and ZrBDC with reproducible properties. In particular, the shaping of the CuBTC and $\mathrm{ZrBDC}$ powders by applying pressure up to 160 and $30 \mathrm{MPa}$, respectively, both in binder-free form and mixed with polyvinyl alcohol (PVA) as a binder, allowed us to prepare the materials with sufficiently high and reproducible performance characteristics. The methane adsorption capacities of thus prepared materials were measured at an experimental ANG bench at 3.5 and $10 \mathrm{MPa}$ and compared with that calculated using the Dubinin theory of volume filling of micropores (TVFM) [59, 60]. The experimental values occurred to be close to the calculation results at high pressure, whereas at 3.5 MPa, a noticeable discrepancy between them was found. The obtained results made it possible to consider the MOF-based materials as promising adsorbents for ANG technology.

Further development of these investigations requires working out optimal conditions for preparing the functional materials from ZrBDC intended for ANG systems. For this purpose, it is necessary to justify the choice of a binder and regime of compaction pressures based on how much the textural properties of the resulting materials change.

In the present study, we examine the factors, which determine the performance of such an ANG system. First, we synthesize chemically and thermally stable $\mathrm{ZrBDC}$ as a basis for preparing functional materials intended for loading the ANG tank (adsorber). Second, we focus on identifying the optimal conditions for producing the MOF-based adsorbent by the simplest way of shaping-mechanical pressing. For this purpose, we compare the results of comprehensive experimental research of textural and methane adsorption properties of the as-prepared ZrBDC powder and ZrBDCbased materials shaped without any binder and with two kinds of the binder: polyvinyl alcohol (PVA) and carboxymethylcellulose (CMC). The changes in the textural properties can be detected by structural investigations, including those performed by such methods as low-temperature nitrogen adsorption, X-ray diffraction (XRD), and scanning electron microscopy (SEM).
Direct measurements of the amount of methane delivered from a mini $\left(100 \mathrm{cmcm}^{3}\right)$ ANG storage tank loaded with the ZrBDC pellets with PVA over a wide temperature range and pressures up to $10 \mathrm{MPa}$ provide a possibility to evaluate the real efficiency of the adsorbent and compare it with the data calculated from the experimental methane adsorption isotherms for the MOF powder and binder-free shaped material. In our work, we measure adsorption of the main NG component-methane, although there are many other impurities $\left(\mathrm{C}_{2}-\mathrm{C}_{5}\right.$ hydrocarbons, carbon dioxide, water, sulfur compounds), which affect the performance of adsorbent in the ANG system [61]. For example, Zhang et al. found that HKUST-1 lost the adsorption capacity by $32 \%$ over 200 sorption/desorption cycles due to irreversible blocking of pores by propane molecules [62]. Although similar cycling investigations in the presence of all NG components are determinative for designing ZrBDC-based materials with the long operational life necessary for their use in an NG-powered vehicle, these questions remained outside the scope of our work.

In the rest of the paper, we concentrated on thermal effects arising during the exothermic/endothermic adsorption/desorption processes, which are also essential for ANG storage performance [63-66]. Indeed, the heat release/absorption effects during the charge/discharge processes change the temperature of the adsorbent, thereby lowering the gas storage performance below the isothermal performance $[63,64]$ and increasing the duration of the charge/discharge processes [65]. On the other hand, the heat of adsorption depends on the strength of the adsorbateadsorbent interactions and is used to get deep insight into the adsorption process [67-69]. Therefore, keeping two goals in mind, we calculate the thermodynamic functions of the $\mathrm{CH}_{4} / \mathrm{ZrBDC}$ material adsorption system from the methane adsorption data in wide ranges of temperatures and pressures for the powdered $\mathrm{ZrBDC}$ and $\mathrm{ZrBDC}$ pellets. First, by evaluating the differential isosteric heat of adsorption, on the one hand, we calculate temperature changes in the ANG storage tank system during the charging process $[65,69]$ and thereby solve a problem of practical relevance. Second, the thermodynamic characteristics of adsorption equilibrium as the entropy, enthalpy, and heat capacity calculated as a function of temperature and amount of adsorbed methane enable us to solve a purely scientific problem, namely, to examine the course of a complex adsorption process, taking into account the changes in the state of adsorbed molecules in pores.

\section{Materials and Methods}

2.1. Synthesis of $\mathrm{ZrBDC}$. ZrBDC was synthesized by the solvothermal method [30] by mixing $0.34 \mathrm{~g}$ BDC $\left(\mathrm{C}_{6} \mathrm{H}_{4}(\mathrm{COOH})_{2}\right)(99+\%$, Acros Organics $)$ in $30 \mathrm{~mL} \mathrm{~N}, \mathrm{~N}$ dimethylformamide (DMF) (chemically pure according to the Russian State Industry Standards or GOST 20289-74, Spectrokhim) with $0.53 \mathrm{~g} \mathrm{ZrCl}_{4}$ (98\%, Acros Organics) in a glass flask. The solution was stirred for $15 \mathrm{~min}$ using a magnetic stirrer without heating. Then, the mixture was placed into an autoclave, and the synthesis was carried out for 
$24 \mathrm{~h}$ at $110^{\circ} \mathrm{C}$. The resulting white precipitate was separated from the initial solution by vacuum filtration and repeatedly washed by hot $\left(\sim 60^{\circ} \mathrm{C}\right) \mathrm{DMF}$. The sample was dried at room temperature for $24 \mathrm{~h}$ and afterward in a ventilated thermostat at $110-160^{\circ} \mathrm{C}$ for $2-5 \mathrm{~h}$.

2.2. Preparation of the ZrBDC-Based Shaped Materials. A hydraulic press was used to prepare the shaped MOFbased materials by compressing the ZrBDC powder in a cylindrical press die mold at the compaction pressure from 30 to $240 \mathrm{MPa}$ for $2 \mathrm{~min}$. The $\mathrm{Zr}$-BDC powder was shaped with no binders (ZrBDC-F) and with polymer binders: a 5\% PVA aqueous solution (ZrBDC-PVA) and a 1\% carboxymethylcellulose sodium salt solution (ZrBDC-CMC). The choice of these binders as optimal was based on the results of the previous study [57]. The MOF:binder mass ratio of the shaped mixture was $1: 1$, which, as shown in [53], allowed one to increase the density and strength of the MOF material with minimal changes in its porous structure (specific surface and micropore volume).

The shaped samples were dried in an oven at $110^{\circ} \mathrm{C}$ for $12 \mathrm{~h}$. The duration and temperature were determined as appropriate from a prior experiment, including periodic weight measurements of a probe sample. The resulting ZrBDC-based samples were cylindrical pellets with a diameter of $38 \mathrm{~mm}$ and a height of $12 \mathrm{~mm}$. These dimensions provided the minimal void space in the ANG vessel (adsorber). The linear dimensions of the pellets were determined by averaging over five measurements according to the standard requirements [70].

Figure 1(a) shows the MOF samples shaped with PVA as a binder-ZrBDC-PVA before loading to the ANG adsorber (b).

Prior to loading the adsorber, the ZrBDC-PVA pellets were regenerated at $160{ }^{\circ} \mathrm{C}$ down to the pressure of $10 \mathrm{~Pa}$.

2.3. Adsorptive. The adsorptive used was high purity (99.999\%) methane purchased from the Moscow Gas Processing Plant (Russia). According to the data reported in [71], methane has the following physicochemical properties: molar mass $\mu=16.0426 \mathrm{~g} / \mathrm{mol}$; critical temperature $T_{\mathrm{cr}}=$ $190.56 \mathrm{~K}$; critical pressure $p_{c r}=4.599 \mathrm{MPa}$. The compressibility and enthalpy of gaseous methane were calculated from the state equations with the virial coefficients provided in the handbook [72].

2.4. Characterization. Elemental composition and surface morphology of the adsorbents were examined by SEM using a Quanta 650 FEG microscope (FEI Company, USA) equipped with an Oxford Energy Dispersive X-ray (EDX) detector. The elemental composition of the sample was determined by averaging ten measurements.

Porous characteristics of the as-prepared and shaped ZrBDC materials were estimated from the adsorption/ desorption isotherm of standard nitrogen vapors at $77 \mathrm{~K}$ measured by a Quantachrome Autosorb iQ multifunctional surface area analyzer. The structural and energy characteristics (SEC), such as the specific micropore volume $W_{0}\left[\mathrm{~cm}^{3} /\right.$ g], standard characteristic energy of nitrogen adsorption $E$
$[\mathrm{kJ} / \mathrm{mol}]$, and average effective radius of micropores $x_{0}$ $[\mathrm{nm}]$, were evaluated by using the theory of volume filling of micropores (TVFM) [59,60]. The specific surface area $\left(S_{\mathrm{BET}}\right)$ of the adsorbents was estimated using the Brunauer-Emmet-Teller (BET) equation [73]. In addition, the specific surface area of mesopores, $S_{\mathrm{ME}}$, was also calculated from the nitrogen adsorption/desorption data at $77 \mathrm{~K}$ using the well-known Kelvin equation [74]. The specific mesopore volume was calculated as $W_{\mathrm{ME}}=W_{\mathrm{S}}-W_{0}$, where $W_{S}$ is the total pore volume calculated from the nitrogen adsorption at the relative pressure $p / p_{s}=0.99$. Here, we have provided the data for the $\mathrm{ZrBDC}$ sample activated at $160{ }^{\circ} \mathrm{C}$ under conditions of thermal-vacuum regeneration. This sample demonstrated the highest SEC values.

XRD patterns of as-prepared ZrBDC were acquired on an Empyrean (Panalytical BV) diffractometer in BraggBrentano geometry using a nickel-filtered $\mathrm{CuK} \alpha$ $\left(\lambda_{\mathrm{Cu}}=0.1542 \mathrm{~nm}\right)$ radiation in the $2 \theta$ angular range of $0^{\circ}$ to $50^{\circ}$.

The packing density of as-prepared ZrBDC powder, $d$, was measured following the standard method [75].

2.5. Methane Adsorption Measurements. High-pressure methane adsorption was measured by the combined volumetric-gravimetric method using the original adsorption setup designed in IPCE RAS [76]. The scheme is provided below in Figure 2 .

Before the measurements, the adsorbent was regenerated by thermal evaporation at $160^{\circ} \mathrm{C}$ to the residual pressure of no more than $1 \mathrm{~Pa}$.

The value of absolute methane adsorption in the MOFbased adsorbents, $a$, was evaluated as follows:

$$
a=\frac{\left(N-\left(V-V_{a}\right) \times \rho_{\mathrm{g}}\right)}{\left(m_{0} \mu\right)} .
$$

Here, $N$ is the amount of methane introduced into the volume of the measuring unit, $\mathrm{g} ; \mathrm{V}$ is the entire volume of the system, $\mathrm{cm}^{3} ; V_{a}$ is the volume of the adsorbent with micropores, $\mathrm{cm}^{3} ; \rho_{g}$ is the density of a gaseous phase at specified pressure $P$ and temperature $T, \mathrm{~g} / \mathrm{cm}^{3} ; m_{0}$ is the mass of regenerated adsorbent, $\mathrm{g} ; \mu$ is the molar mass of methane, $\mathrm{g} /$ mmol. The value of $V_{a}$ was calculated as a sum of the adsorbent skeleton volume determined from helium picnometry measurements, $V_{\mathrm{He}}$, and the product $m_{0} \times W_{0}$. The value of $W_{0}$ was calculated from the standard low-temperature nitrogen adsorption data by TVFM equations [59, 60].

The accuracy of methane adsorption measurements was $\pm 5 \%$ at a confidence level of 0.95 for most experimental values. The uncertainty of adsorption measurements was calculated following the standard requirements [70].

The deliverable volumetric methane capacity of the shaped MOF, i.e., the amount of methane delivered from an ANG vessel when the pressure dropped from 10 to $0.1 \mathrm{MPa}$, was measured on an ANG bench according to the recently reported route [77] (see Figure 3).

The main element of the scheme is a stainless steel ANG tank or adsorber (1), see Figure 1(b), with a volume of 


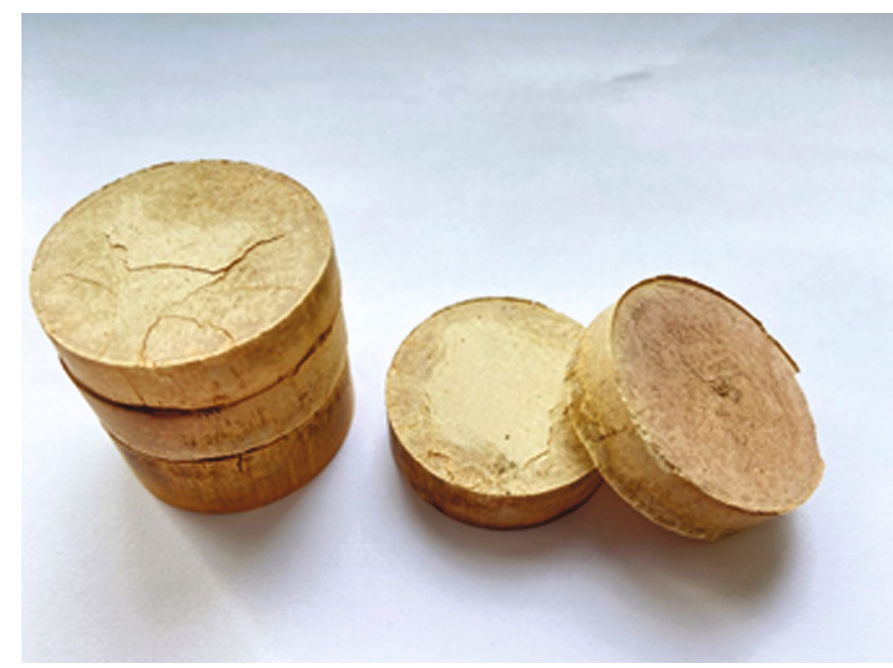

(a)

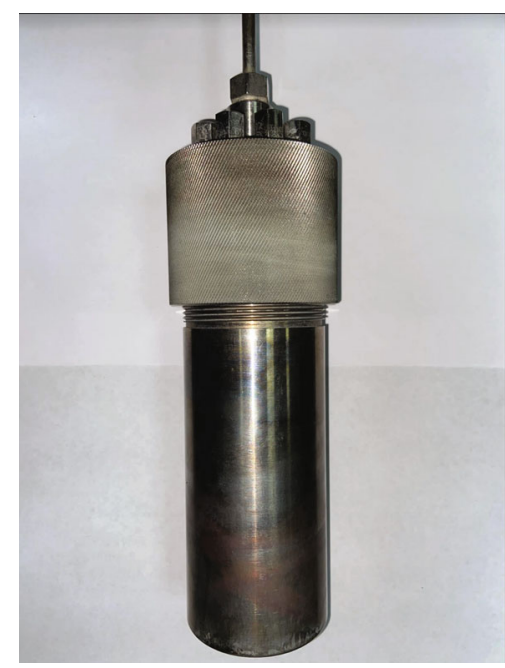

(b)

FIgure 1: Photographs of the ZrBDC-PVA pellets (a) prepared by compacting the ZrBDC powder under the shaping pressure of 30 MPa at the temperature of $298 \mathrm{~K}$ for loading into the ANG tank (b).

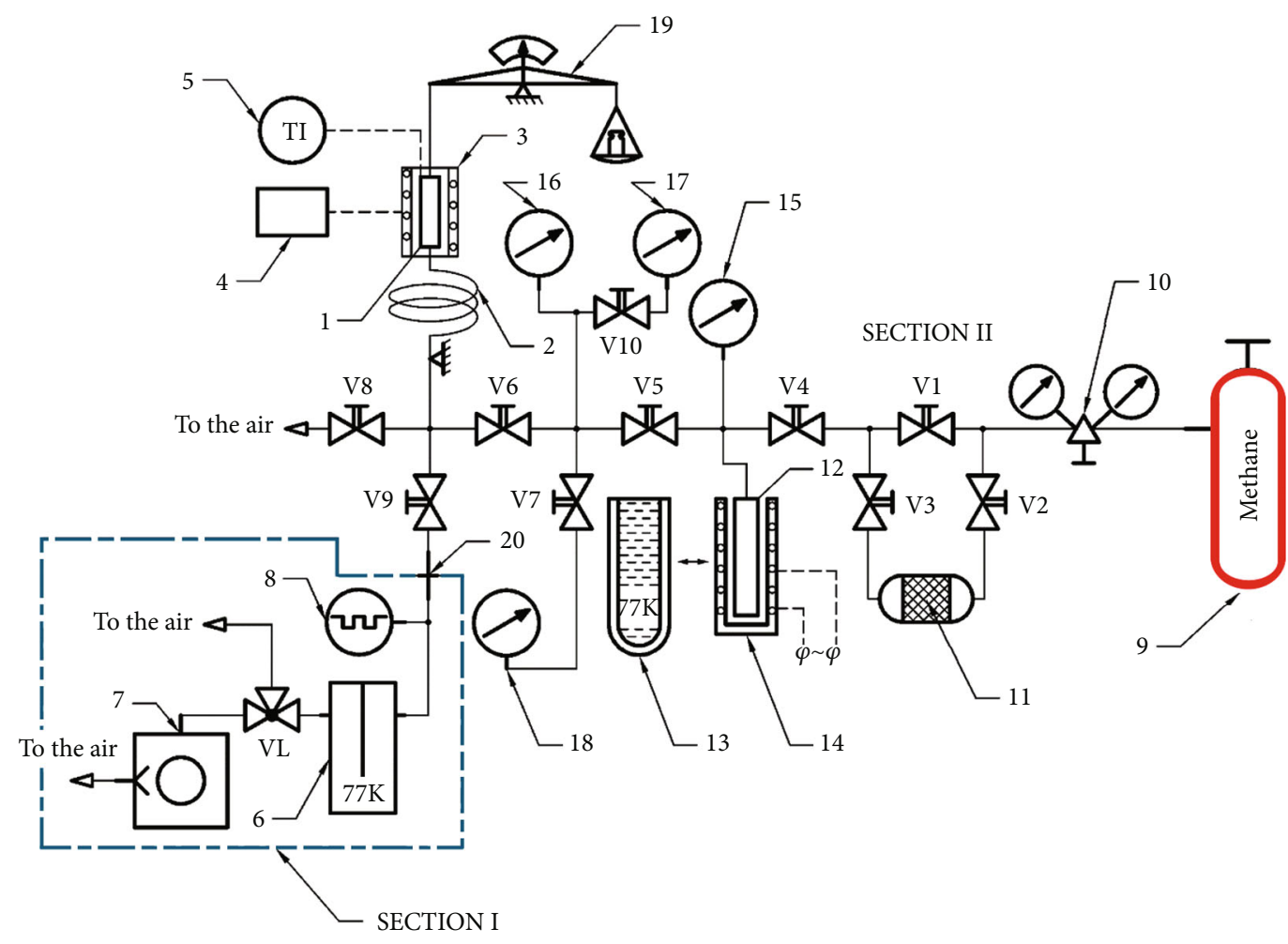

Figure 2: Schematic representation of the high-pressure adsorption setup for methane adsorption measurements by the combined gravimetric-volumetric method [66]: I-high-pressure section made of metal; II-vacuum section made of glass; 1-ampoule with an adsorbent; 2-capillary tube for supply with methane; 3-thermostated air bath; 4-temperature controller; 5-temperature acquisition; 6nitrogen trap; 7-fore vacuum pump; 8-ionization thermocouple vacuum manometer; 9-methane cylinder; 10-pressure reducer; 11 methane cleanup unit; 12-thermal compressor; 13-Dewar vessel; 14-heating unit; 15-18-manometer; 19-comparative beam scales; 20"kovar-glass" junction of Sections I and II; V1-V10: valves; VL: vacuum lock.

$100 \mathrm{~cm}^{3}$ loaded with the ZrBDC MOF-based pellets. The ANG tank is immersed in the thermostatically controlled chamber (2) filled with a $96 \%$ aqueous ethanol solution. The chamber is equipped with a platinum resistive tempera- ture transducer that serves as a temperature indicator TTI and a temperature regulator-TRC. The temperature was maintained within an accuracy of $\pm 0.1 \mathrm{~K}$. The gas unit, consisting of a methane source (3), a low-pressure receiver (4), a 


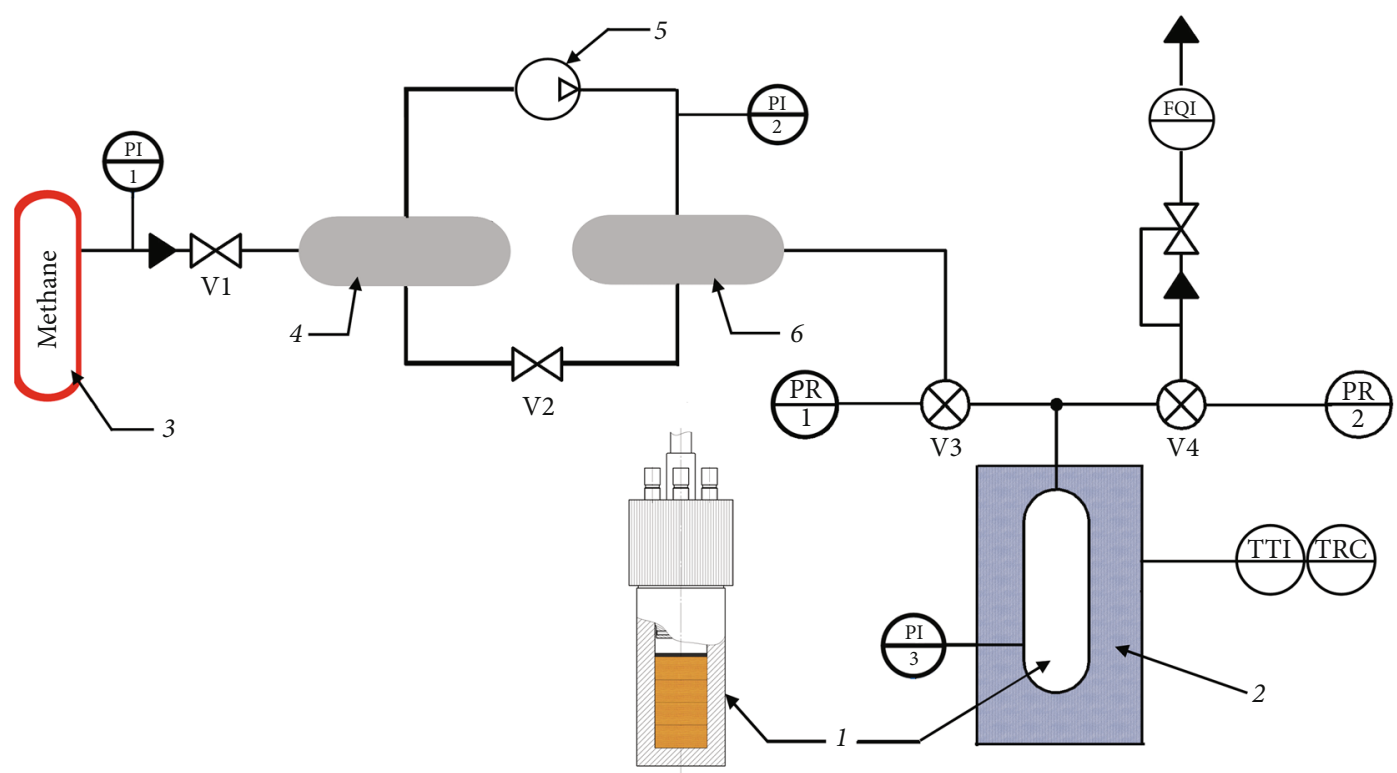

FIGURE 3: Schematic representation of the experimental ANG bench for direct measurements of adsorbed methane storage: 1-ANG storage vessel (adsorber) loaded with the shaped MOF-based pellets; 2-thermostatically controlled chamber filled with a coolant/heat carrier medium; 3-methane source; 4-low-pressure receiver; 5-compressor; 6-high-pressure receiver; PI1-3: pressure indicators; PR1-2: pressure regulator valves; V1-4: gas lock valves; TTI: temperature detector; TRC: temperature adjustment unit; FQI: gas counter.

compressor (5), and a high-pressure receiver (6), supplied the adsorber with methane. The ANG bench involves several gas-lock valves V1-4 connected by the high-pressure pipelines. With valve V4 closed, valves V1-V3 were opened, and methane was pumped to the adsorber up to a required pressure $(1,3.5,7$, and $10 \mathrm{MPa})$. The pressure regulators PR1-2 ensure the required level of pressure with an accuracy of $\pm 0.15 \%$. After achieving the equilibrium in the system, valves V1-V3 were closed, V4 was opened, and the delivery was started. The process was carried out at a rate of about $10 \mathrm{~L} / \mathrm{min}$ until the excess pressure of $10,7,3.5$, and $1 \mathrm{MPa}$ decreased to $0.1 \mathrm{MPa}$. The FQI gas counter readings were taken only after reaching equilibrium pressure and temperature. The relative error in measuring the amount of gas by FQI was $\pm 1 \%$.

To take into account a dead volume, before the measurements, the system was calibrated at the same thermodynamic conditions using a mockup (MO) mounted inside the ANG vessel. The mockup volume was equal to that of the stack of the ZrBDC pellets. The correction to the deliverable storage capacity was calculated as the difference between the FQI readings: $V_{\mathrm{FQI}-\mathrm{ANG}}$ and $V_{\mathrm{FQI}-\mathrm{MO}}$.

Before the measurements, three cycles of methane displacement adsorption up to $25 \mathrm{MPa}$ were carried out to prepare the bench.

\section{Results and Discussion}

3.1. SEM Analysis of as-Synthesized ZrBDC. As follows from the SEM images at various magnifications (Figures 4(a) and 4(b)), the surface of as-prepared ZrBDC is formed by the interlocking of cubic micron-sized particles.

As follows from Table 1, which summarizes the SEMEDX data, the chemical composition of the ZrBDC sample is determined by the reagents used in the synthesis. It can be seen that carbon is the dominant element in the sample. The presence of $\mathrm{O}, \mathrm{Cl}$, and $\mathrm{Zr}$ atoms in the pores of $\mathrm{ZrBDC}$ can contribute to the methane adsorption, enhancing the $\mathrm{CH}_{4}-\mathrm{ZrBDC}$ interactions. Although, as shown by Kumar et al. [78] and Vandenbrande et al. [79], the narrowest constricted micropores are the preferential adsorption sites of methane in UiO-type MOFs. In contrast, $\mathrm{Zr}$ ions do not contribute any significant energy heterogeneity within the pores for methane adsorption. Moreover, the inorganic building units can hinder the access of methane to the $\mathrm{Zr}$ clusters [78].

3.2. The Density of the Shaped ZrBDC-Based Adsorbents. Figure 5 shows the densities of the shaped ZrBDC samples prepared without any binder and mixed with CMC and PVA versus the compaction pressure. For comparison, the star symbol in the plot marks the packing density of assynthesized powder ZrBDC (4).

As follows from Figure 5, the packing density of the shaped samples increases with the compaction pressure. The most significant increase in the density from 0.3 to $1.6 \mathrm{~g} / \mathrm{cm}^{3}$ was achieved upon the shaping process without any binder (see curve 1 for ZrBDC-F) at the compaction pressure of $240 \mathrm{MPa}$. Under the same conditions, the compaction of ZrBDC mixed with PVA or CMC increased the density of the final materials by about 3.7 times (from 0.3 to $1.1 \mathrm{~g} / \mathrm{cm}^{3}$ ). It should be noted that when the applied pressure varied from 30 to $50 \mathrm{MPa}$, the densities of the shaped materials could be categorized into ascending order: $\mathrm{ZrBDC}-\mathrm{F}<\mathrm{ZrBDC}-\mathrm{CMC}<\mathrm{ZrBDC}-\mathrm{PVA}$, but when the pressure increased from 70 to $240 \mathrm{MPa}$, it transformed into descending order as follows: $\mathrm{ZrBDC}-\mathrm{F}>\mathrm{ZrBDC}-$ $\mathrm{PVA}>\mathrm{ZrBDC}-\mathrm{CMC}$. 


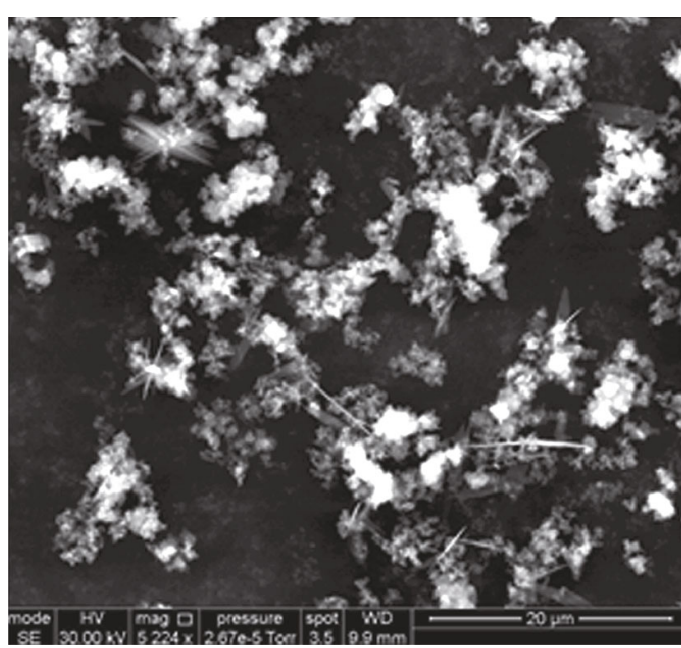

(a)

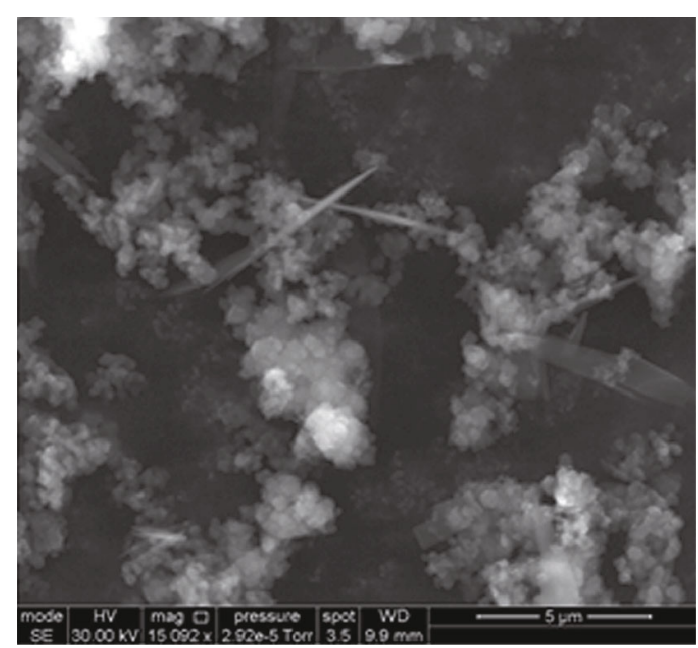

(b)

FIgURE 4: SEM images of the as-prepared ZrBDC sample. The scale bars are 20 (a) and $5 \mu \mathrm{m}$ (b).

TABLE 1: Elemental chemical composition of ZrBDC evaluated from the SEM-EDX data and represented in weight (wt.\%) and atomic (at.\%) percentage.

\begin{tabular}{lcc}
\hline Element & Wt. $\%$ & At. $\%$ \\
\hline $\mathrm{C}$ & 27 & 50 \\
$\mathrm{Cl}$ & 4 & 3 \\
$\mathrm{O}$ & 26 & 37 \\
$\mathrm{Zr}$ & 43 & 10 \\
\hline
\end{tabular}

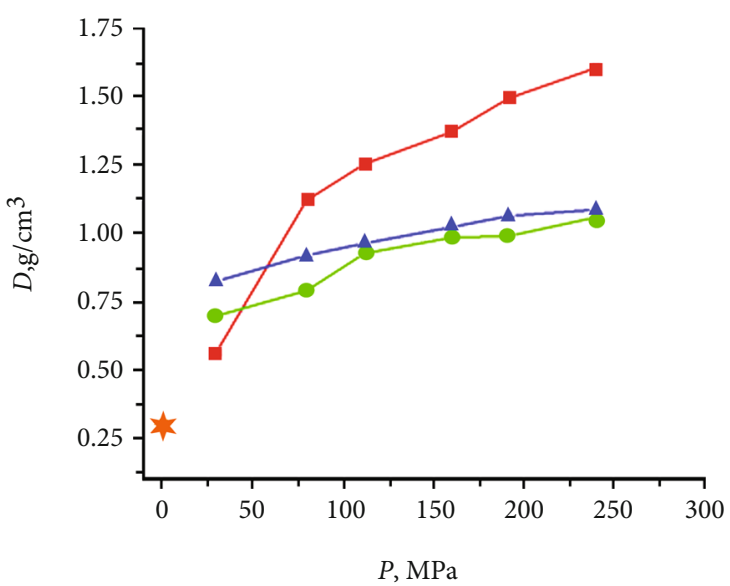

FIgURE 5: Density of the ZrBDC-based materials prepared without any binders, ZrBDC-F (1), and mixed with the binders: ZrBDCPVA (2) and ZrBDC-CMC (3), versus the compaction pressure. Orange star denotes the packing density of as-prepared powder ZrBDC. The temperature of measurements was $298 \mathrm{~K}$.

3.3. Changes in the Porous Structure of the ZrBDC-Based Materials upon Shaping. It is obvious that the compaction of the as-synthesized ZrBDC powder removed the interparticle voids resulting in the more close packing of the particles observed in Figure 4. The effect of compaction on the microand mesoporous structure of $\mathrm{ZrBDC}$ was revealed by com- paring the parameters of the porous structure calculated from the isotherms of low-temperature nitrogen vapor adsorption in the as-synthesized ZrBDC powder and the shaped materials. Figures $6(\mathrm{a})$ and $6(\mathrm{~b})$ demonstrate the changes in the porous structure of the ZrBDC-based materials with compaction at $298 \mathrm{~K}$.

As follows from the data in Figure 6(a), the compaction of the ZrBDC powder without a binder produces a noticeable degradation of its porous structure: when the compaction pressure was $240 \mathrm{MPa}$, the specific BET surface decreases almost threefold: from 1100 to $340 \mathrm{~m}^{2} / \mathrm{g}$. The recent XRD experiments have illustrated the degradation of the ZrBDC structure upon its compaction under different pressures [58]. Thus, one can expect that the high-pressure shaping of ZrBDC results in a significant loss in adsorption capacity for methane. However, when the compaction pressures are relatively low, $30-50 \mathrm{MPa}$, the resulting materials ZrBDC-F and ZrBDC-PVA retain the highly developed porous structure. This fact is illustrated by Table 2, which lists the porous structure parameters of the ZrBDC-based materials before and after the compaction at $30 \mathrm{MPa}$ in the binder-free form and mixed with PVA and CMC. As is evident from Table 2, the compaction of ZrBDC with PVA at $30 \mathrm{MPa}$ led to the increase in the density by more than two times, which was accompanied by a $13.6 \%$ reduction in the micropore volume and a $6.5 \%$ reduction in the specific surface area. Thus, we obtained an adsorbent, ZrBDC-PVA, with the lowest degradation of the porous structure compared to CMC. Moreover, note that the superiority in the density observed for ZrBDC-CMC is negligible compared to $\mathrm{ZrBDC}-\mathrm{PVA}$.

Here, $E_{0}$ is the characteristic energy of standard benzene vapors calculated from the ratio $E_{0}=E / \beta$, where $\beta=\Pi / \Pi_{0}$ $=0.34$ is the affinity coefficient determined as the ratio of parachors of the studied vapor (nitrogen) $\Pi$ to standard benzene vapor $\Pi_{0}$ at the boiling points [60].

The comparative analysis of the textural properties of the as-prepared ZrBDC powder and shaped materials in Table 2 


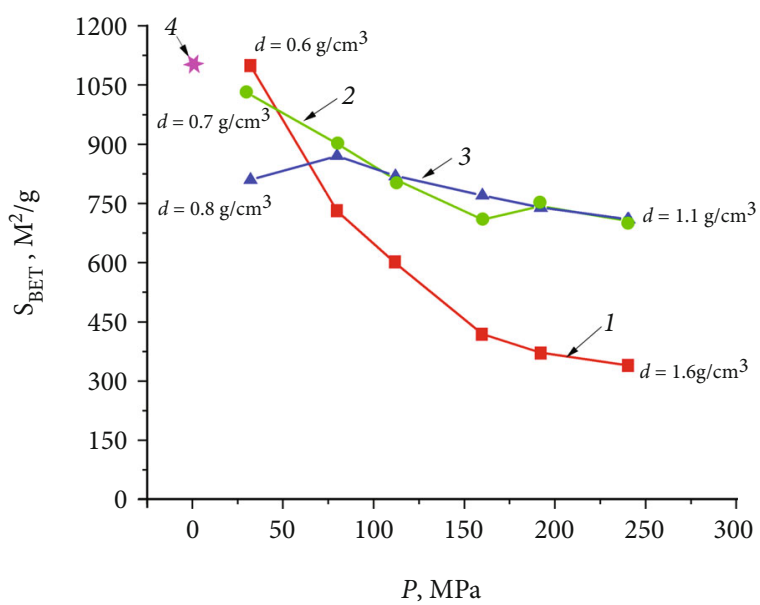

(a)

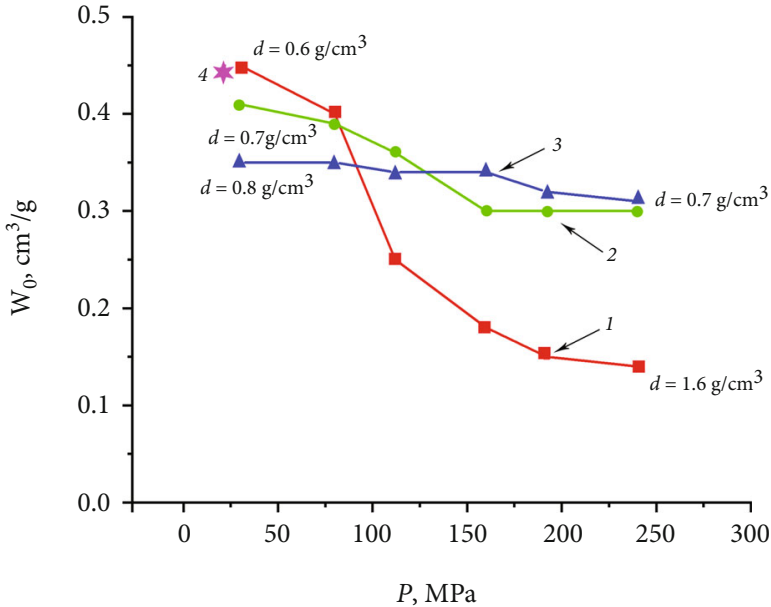

(b)

FIGURE 6: Specific surface area $S_{\mathrm{BET}}$ (a) and micropore volume $W_{0}$ of the ZrBDC-based materials: ZrBDC-F (1), ZrBDC-PVA (2) and ZrBDC-CMC (3), versus the compaction pressure. The star symbol (4) shows the values of $S_{\mathrm{BET}}$ and $W_{0}$ of the as-prepared ZrBDC powder.

TABLE 2: Parameters of the porous structure and the packing density of the as-prepared ZrBDC powder and shaped materials: ZrBDC-F, ZrBDC-PVA, and ZrBDC-CMC prepared under the compaction pressure of $30 \mathrm{MPa}$ and temperature of $298 \mathrm{~K}$.

\begin{tabular}{lcccccccc}
\hline Sample & $W_{0}, \mathrm{~cm}^{3} / \mathrm{g}$ & $E_{0}, \mathrm{~kJ} / \mathrm{Mol}$ & $x_{0}, \mathrm{~nm}$ & $E, \mathrm{~kJ} / \mathrm{Mol}$ & $S_{\mathrm{BET}}, \mathrm{m}^{2} / \mathrm{g}$ & $W_{S}, \mathrm{~cm}^{3} / \mathrm{g}$ & $W_{\text {me }}, \mathrm{cm}^{3} / \mathrm{g}$ & $d, \mathrm{~g} / \mathrm{cm}^{3}$ \\
\hline ZrBDC & 0.44 & 22.4 & 0.54 & 7.4 & 1100 & 0.67 & 0.13 & 0.30 \\
ZrBDC-F & 0.44 & 20.8 & 0.58 & 6.9 & 1100 & 0.54 & 0.09 \\
ZrBDC-PVA & 0.38 & 23.6 & 0.51 & 7.4 & 930 & 0.54 & 0.16 \\
ZrBDC-CMC & 0.35 & 25.1 & 0.54 & 8.3 & 810 & 0.36 & 0.01 \\
\hline
\end{tabular}

revealed the following facts, which are essential for adsorption:

(i) The compaction of the as-prepared ZrBDC powder at 30-50 MPa results in a decrease in the total pore volume $W_{S}$ due to the destruction of mesopores. At the same time, ZrBDC-F demonstrates the lowest value of characteristic energy of adsorption (benzene) $E_{0}$ and, consequently, the widest micropores among the materials. A larger proportion of micropores in the total pore volume of ZrBDC-F compared to other shaped materials causes the highest specific BET surface and the lowest characteristic energy of adsorption

(ii) The binders used in the shaping process increased the proportion of micro- and mesopores in the total pore volume of the final ZrBDC-based materials. The relatively low values of the micropore width and volume in ZrBDC-PVA indicated the penetration of the PVA molecules mainly into micropores. In contrast, CMC molecules with a larger size than PVA [80] were able to enter only mesopores, and hence the lowest mesopore volume was found for ZrBDC-CMC, whereas the micropore width in this material coincided with that observed for the as-prepared ZrBDC. As follows from the data for ZrBDC-PVA, the PVA molecules were not able to block mesopores due to the smaller molecular sizes [81] (iii) Among the three shaped MOFs, two materials can be considered promising adsorbents for ANG systems [2]. The relatively developed porous structure of $\mathrm{ZrBDC}-\mathrm{F}$ is expected to provide its significant adsorption capacity for methane, whereas the high packing density of ZrBDC-PVA ensures the high performance for methane storage

3.4. XRD Analysis of ZrBDC-Based Materials. The main reflections at $2 \theta \sim 7,8,12$, and $25^{\circ}$ on the XRD pattern for the as-prepared ZrBDC (Figure 7, curve 1) coincide with that calculated for UiO-66 [49]. In the previous work [57], the XRD data for ZrBDC powder compacted without any binders under different pressures revealed that the main reflections became less pronounced as the applied compaction pressure increased, indicating a progressive degradation of the ZrBDC-PVA crystalline structure.

The comparison of XRD patterns recorded before and after the shaping of the as-prepared ZrBDC powder with PVA and CMC (see Figure 7) enabled us to observe the effects of the binders on the MOF structure upon their compaction at $160 \mathrm{~Pa}$.

The insignificant changes in the XRD patterns of the ZrBDC shaped with PVA and CMC lead us to conclude that the binder helps minimize the destruction of the MOF structure during the shaping process. It should be noted that both binders produced almost the same "protective" effect for the ZrBDC crystalline structure. Nevertheless, PVA was chosen 


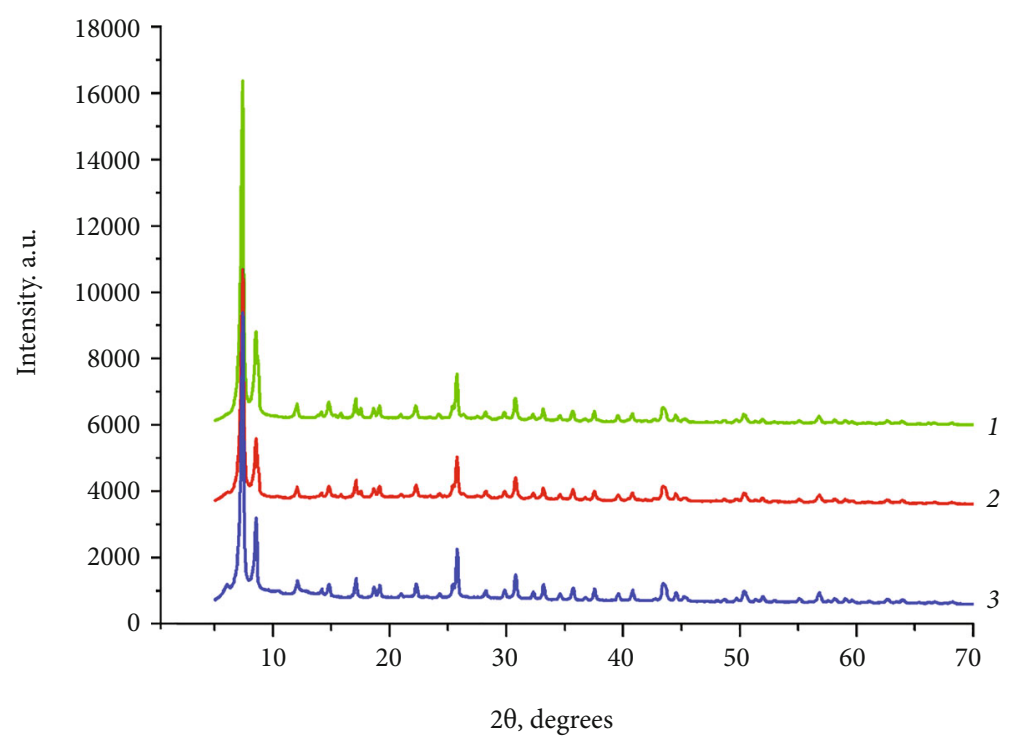

FIGURE 7: XRD patterns of as-prepared ZrBDC powder before (1) and after shaping with PVA (2) and CMC (3) as a binder under the shaping pressure of $160 \mathrm{MPa}$.

as the most optimal binder for preparing monolithic ZrBDC-based materials on the ground of the cumulative contribution of a binder to the density, porous, and crystalline structures of the resulting MOF upon the shaping process (Table 2, Figures 5, 6(a), and 6(b)),

3.5. Methane Adsorption in ZrBDC-Based Materials. Figure 8 shows the isotherms of methane adsorption on the as-prepared $\mathrm{ZrBDC}$ sample measured up to the pressure of $10 \mathrm{MPa}$ within a range of temperatures from 253 to $333 \mathrm{~K}$. All isotherms show no adsorption-desorption hysteresis loop, indicating a reversible process of physical adsorption. The experimental isotherms were fitted by a formula derived by Bakaev using statistical thermodynamics for a model of adsorption in cavities considered as quasi-independent subsystems of the grand canonical ensemble [82]:

$$
a(P)=\frac{k_{0}\left(k_{1} P+2 k_{2} P^{2}+3 k_{3} P^{3}\right)}{1+k_{1} P+k_{2} P^{2}+k_{3} P^{3}},
$$

where $k_{0}$ characterizes an adsorption system, $k_{1}, k_{2}$, and $k_{3}$ are the temperature-dependent and numerically adjusted coefficients, and $P$ is the equilibrium pressure expressed in $\mathrm{Pa}$.

This formula has been successfully applied to describe the experimental adsorption isotherms of different gases on zeolites [82], polymer adsorbents [83], and activated carbons $[84,85]$. In our experiments, the regression error was not more than $2 \%$.

To assess the effectiveness of an ANG storage system, the total specific volumetric capacity of the system, $V_{F}$, is employed. This parameter involves the amount of adsorbed gas and the amount of gas in the intergranular space outside the adsorbent:

$$
V_{F}(P, T)=\frac{a(P, T) \mu d+\varepsilon \rho_{\mathrm{g}}(P, T)}{\rho_{\mathrm{NTP}}},
$$

where $\rho_{\mathrm{g}}$ is the density of a free gaseous phase in an ANG tank $\left(\mathrm{kg} / \mathrm{m}^{3}\right)$ at the specified pressure $P$ and temperature $T$ ; $d$ is the packing density of the adsorbent $\left(\mathrm{kg} / \mathrm{m}^{3}\right), \mathrm{NTP}$ is the standard thermodynamic conditions for gases: $T=$ $293.15 \mathrm{~K}, P=101.325 \mathrm{kPa}, \rho_{\mathrm{NTP}}$ is the density of the gas at the NTP conditions $\left(\mathrm{kg} / \mathrm{m}^{3}\right)$, and $\varepsilon$ is the adsorbent bed void fraction in the ANG tank, which was calculated as follows:

$$
\varepsilon=1-d\left(W_{0}+\frac{1}{d_{c}}\right)
$$

Here, $d_{c} \approx 1650 \mathrm{~kg} / \mathrm{m}^{3}$ is the skeleton density of ZrBDC [26].

The deliverable volumetric capacity of the ANG system, $V_{A}$, is more important for evaluating its performance since it determines the driving range of NG vehicles. The value of $V_{A}$ is evaluated as the amount of gas at standard thermodynamic conditions, which can be delivered from the tank with adsorbent upon isothermal gas discharge from the current level of pressure $P$ in the tank to the minimal value $P_{x}$ (in our case, $P_{x}=0.1 \mathrm{MPa}$ ) normalized to the geometric volume of the tank:

$$
V_{A}=V_{F}(P, T)-V_{F}\left(P_{0.1}, T\right) .
$$

Figures $8(\mathrm{a})$ and $8(\mathrm{~b})$ show the total specific volumetric capacities of the adsorption systems with the ZrBDC (a) and ZrBDC-F (b) materials used as adsorbents represented as a function of pressure within the temperature range of $253-333 \mathrm{~K}$. The data were calculated from the experimental isotherms of methane adsorption according to Eq. (3) and 


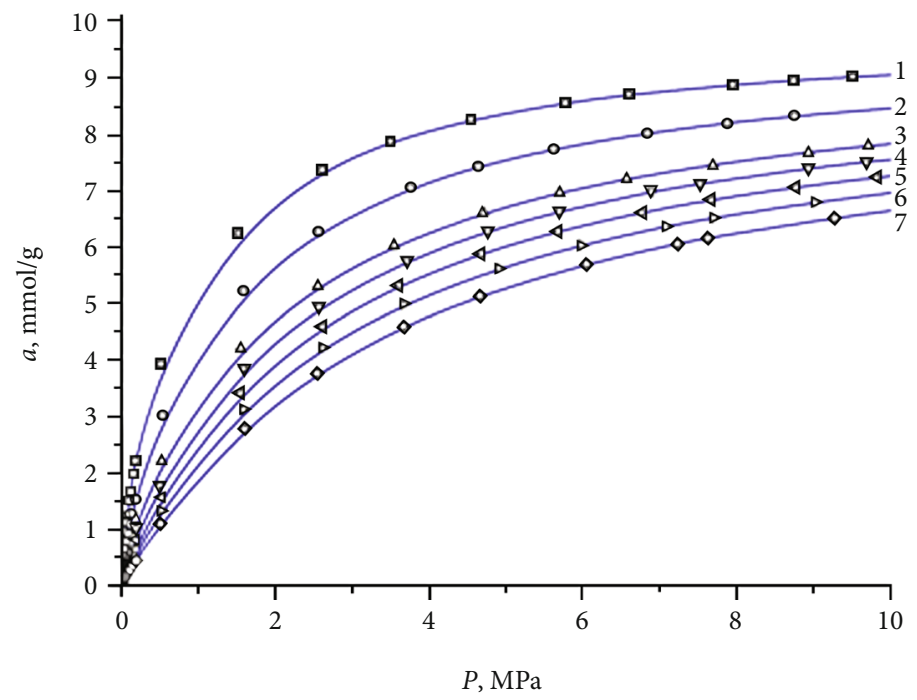

FIgURE 8: Methane adsorption isotherms for as-prepared ZrBDC at temperatures. K: 253 (1), 273 (2), 293 (3), 303 (4), 313 (5), 323 (6), and 333 (7). Symbols show the experimental data, and the solid lines are the results of approximation by Eq. (2).

compared with the specific volumetric capacity of a $\mathrm{CNG}$ system calculated for the same thermodynamic conditions (dashed lines in Figure 9).

It was found that the total specific volumetric capacity of the ANG system with ZrBDC achieved $175 \mathrm{~m}^{3}(\mathrm{NTP}) / \mathrm{m}^{3}$ at $293 \mathrm{~K}$ and $10 \mathrm{MPa}$ (Figure $9(\mathrm{a})$ ). This value occurred to be less than that for the ANG system with ZrBDC-F, which exceeded $190 \mathrm{~m}^{3}(\mathrm{NTP}) / \mathrm{m}^{3}$ (Figure $9(\mathrm{~b})$ ). The reason is an almost twofold increase in the packing density of the adsorbent due to the compaction (see Table 2). Moreover, the regime of the maximum absolute efficiency of the ANG system with $\mathrm{ZrBDC}-\mathrm{F}$, i.e., when $V_{F}^{\mathrm{ANG}}-V_{F}^{\mathrm{CNG}}>0$, is observed within the pressure range from 1 to $6 \mathrm{MPa}$ and is almost double this parameter for the system with ZrBDC.

A similar tendency is also observed for the relationship between the specific volumetric deliverable capacities of the ANG systems with as-synthesized powder ZrBDC and compacted binder-free ZrBDC-F (compare Figures 10(a) and $10(\mathrm{~b})$ ). It should be noted that at $253 \mathrm{~K}$ and when the pressures are above $10 \mathrm{MPa}$, the values of $V_{A}{ }^{A N G}$ for the ANG system with $\mathrm{ZrBDC}$ are less than $V_{A}{ }^{\mathrm{CNG}}$. Figure 10 (c) shows the values of $V_{A}$ ANG obtained on the experimental ANG bench with the adsorber loaded with ZrBDC-PVA pellets. The data on $V_{A}$ ANG are represented as a function of pressure at four temperatures: 253, 273, 293, and $303 \mathrm{~K}$.

It was found that at $10 \mathrm{MPa}$ and $253 \mathrm{~K} V_{A}$ achieved the value of $172 \mathrm{~m}^{3}(\mathrm{NTP}) / \mathrm{m}^{3}$ for the $\mathrm{ANG}$ system with ZrBDC-PVA and $155 \mathrm{~m}^{3}(\mathrm{NTP}) / \mathrm{m}^{3}$ for ZrBDC. Note that at the same $P, T$-conditions, the ANG system with the ZrBDC-F adsorbent shows the deliverable capacity of 160 $\mathrm{m}^{3}(\mathrm{NTP}) / \mathrm{m}^{3}$, whereas this value exceeds that for ZrBDCPVA by $3-5 \%$ within the range of pressures from 2 to $6 \mathrm{MPa}$. However, the relatively poor mechanical properties of $\mathrm{ZrBDC}-\mathrm{F}$ will limit its practical application. Indeed, the Shore hardness measurements [86] carried out for the shaped ZrBDC-PVA, ZrBDC-CMC, and ZrBDC-F materials gave the values of 95, 96.5, and 50 Shore A units, respectively.

As shown above, the compaction of the ZrBDC powder, on the one hand, increased its density, but, on the other hand, the parameters of the porous structure deteriorated. These factors had opposite effects on methane adsorption. As follows from the obtained results, the ZrBDC-PVA pellets prepared under the pressure of $30 \mathrm{MPa}$ are characterized by increased density and partially degraded porosity, but they demonstrated higher performance than the MOF powder. The compaction at $30 \mathrm{MPa}$ with PVA improved the deliverable methane capacity of ZrBDC at $10 \mathrm{MPa}$ and $253 \mathrm{~K}$ by $\sim 11 \%$.

\subsection{Thermodynamic Properties of the CH4/ZrBDC Adsorption System}

3.6.1. Thermodynamic State Functions. As known [63-66, 69], the effectiveness of the ANG system is determined not only by the adsorption properties of the porous material but also by the heat effects arising during the methane adsorption/desorption processes. The adsorbent bed in the ANG tank releases heat during the charging process. In contrast, it gets cool during discharge. In addition, if the temperature in the adsorber exceeds the maximum endurance temperature, then the adsorber would not be able to withstand the pressure, and there may be a release of the fuel. Thermal management of an ANG system (heat exchangers, material construction, and geometry of the adsorber) requires the knowledge of thermodynamic characteristics of the adsorption system, namely, the heat of adsorption. The latter provided a rationale to evaluate the thermodynamic state functions of the investigated adsorption systems.

The differential molar isosteric heat of adsorption, $q_{\mathrm{st}}$, is calculated as the difference between the molar enthalpy of 


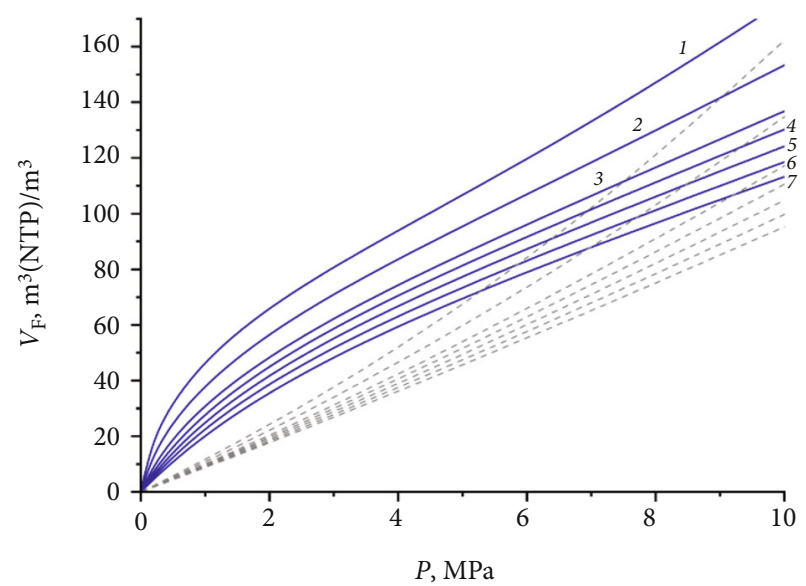

(a)

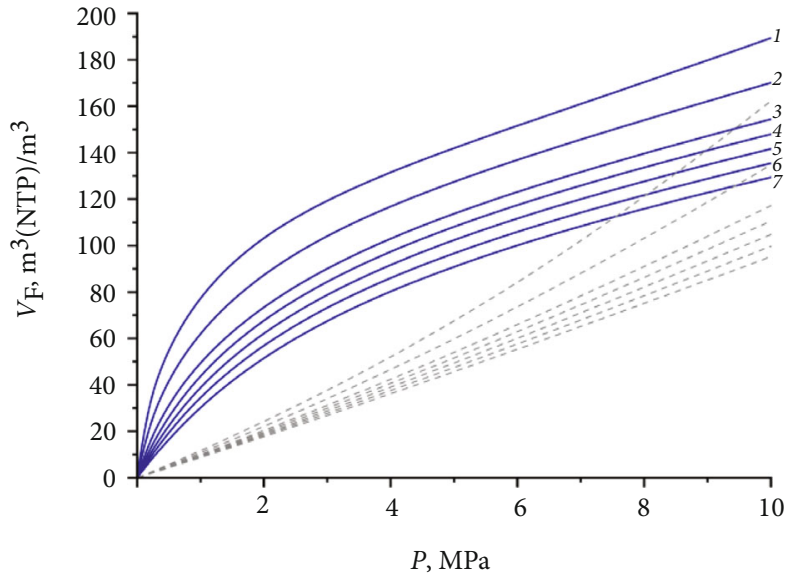

(b)

Figure 9: Total specific volumetric methane capacities calculated for the ANG system (solid lines) with ZrBDC (a) and shaped ZrBDC-F (b) versus methane pressure at the temperature, K: 253 (1), 273 (2), 293 (3), 303 (4), 313 (5), 323 (6), and 333 (7). Dashed lines show the dependences for a CNG system at the same $P, T$-conditions.

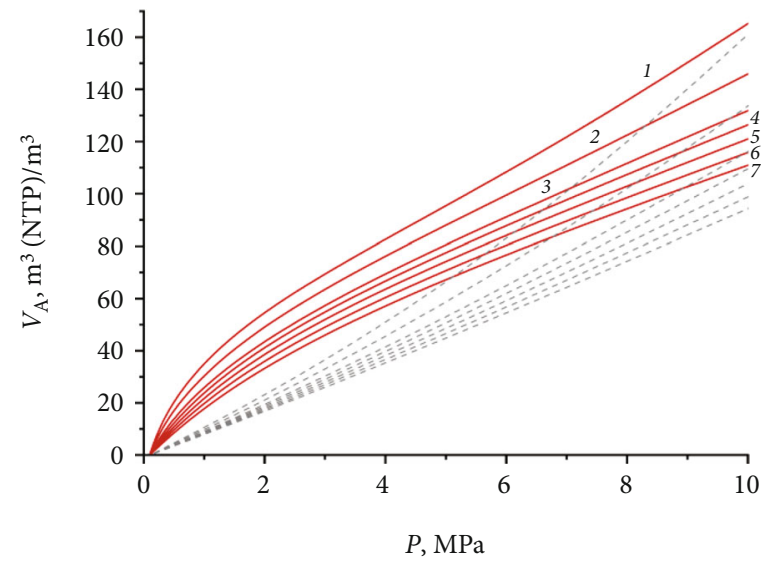

(a)

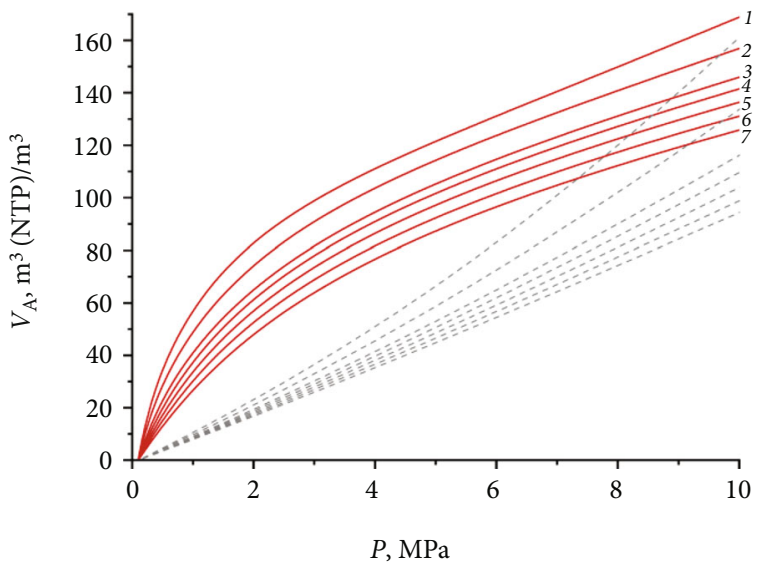

(b)

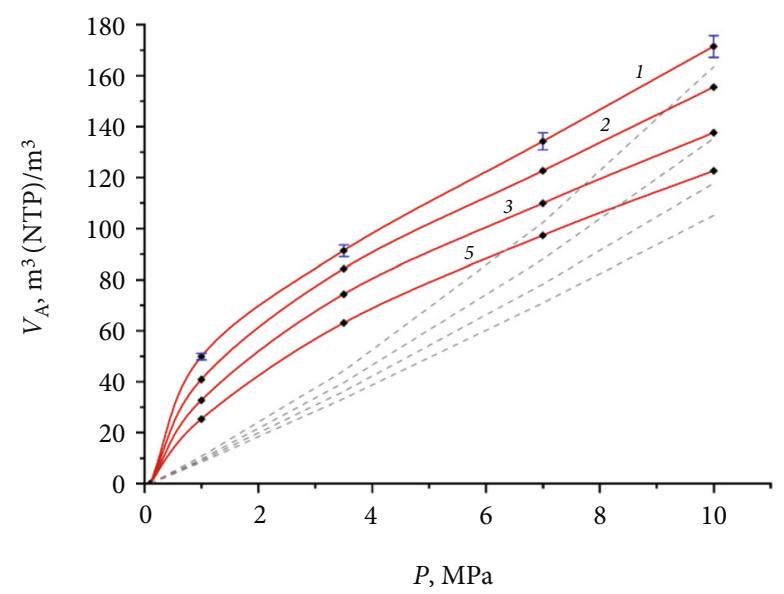

(c)

FIGURE 10: Deliverable specific volumetric methane capacities for the ANG system with as-synthesized powder ZrBDC (a), shaped ZrBDC-F (b), and ZrBDC-PVA (c) versus pressure at the temperature, K: 253 (1), 273 (2), 293 (3), 303 (4), 313 (5), 323 (6), and 333 (7). Solid lines in (a) and (b) show the results of calculations by Eq. (5) using the methane adsorption data. Symbols in (c) show the results of measurements on the experimental ANG bench with the adsorber loaded with the ZrBDC-PVA pellets; solid lines in (c) are spline-approximation. Dashed lines show the dependence for a CNG system. The error bars are 5\%. 
an equilibrium gas phase, $h_{g}$, and the differential molar enthalpy of an adsorption system, $h_{1}$ [87]:

$$
q_{s t}=h_{g}-\left(\frac{\partial H_{1}}{\partial a}\right)_{T}=h_{g}-h_{1} .
$$

Here, $H_{1}$ is the enthalpy of the adsorption system.

We employed an approach developed by Bakaev [88] and reported in details in $[66,89]$ for calculating the differential molar isosteric heat of adsorption from the experimental isosteres of absolute methane adsorption with consideration of the nonideality of the gaseous phase and noninertness of the adsorbent:

$$
q_{s t}=-R \cdot Z\left[\frac{\partial(\ln P)}{\partial(1 / T)}\right]_{a} \cdot\left[1-\left(\frac{\partial V_{a}}{\partial a}\right) / v_{g}\right]-\left(\frac{\partial P}{\partial a}\right)_{T}\left(V_{a}-T \frac{\partial V_{a}}{\partial T}\right)_{a} .
$$

Here $Z=\mathrm{Pv}_{g} / \mathrm{RT}$ is the compressibility of the equilibrium gaseous phase at the specified $P, T$-conditions $(\mathrm{Pa}, \mathrm{K}) ; v_{g}$ is the specific volume of the gaseous phase, $\mathrm{m}^{3} / \mathrm{kg}$; $R$ is the universal gas constant, $\mathrm{J} /(\mathrm{mol} \cdot \mathrm{K}) ; V_{a}=V_{0}(P, T) / m_{0}$ is the specific reduced volume of the adsorption system, $\mathrm{cm}^{3} / \mathrm{g}$; $V_{0}(P, T)$ is the volume of the adsorbent including micropores; $m_{0}$ is the mass of regenerated adsorbent.

Thus, formula (7) includes the term associated with the isothermal adsorption-induced deformation $\left(\partial V_{a} / \partial a\right)_{T}$ and isosteric deformation $\left(\partial V_{a} / \partial T\right)_{a}$ of the adsorbent, the slope of the adsorption isotherms $(\partial P / \partial a)_{T}$ and isosteres $[\partial \ln P / \partial(1 / T)]_{a}$, and the nonideality of the gaseous phase $Z$ $(P, T)$.

When the effects associated with thermal and adsorption noninertness (deformation) of an adsorption system are insignificant, Eq. (7) simplifies to

$$
q_{\mathrm{st}}=R \cdot Z\left[\frac{\partial(\ln P)}{\partial(1 / T)}\right]-\left(\frac{\partial P}{\partial a}\right)_{T} \cdot V_{a}
$$

It should be noted that ZrBDC-based MOF is considered exceptionally rigid and thermally stable due to the high degree of coordination of the $\mathrm{Zr}-\mathrm{O}$ metal centers to the organic linkers [90]. Therefore, the adsorption- and temperature-stimulated deformations of the $\mathrm{ZrBDC}$ material, $\left(\partial V_{a} / \partial a\right)_{T}$ and $\left(\partial V_{a} / \partial T\right)_{a}$ could be neglected when calculating the differential isosteric heat of adsorption.

The heat of adsorption is determined by the adsorbateadsorbent and adsorbate-adsorbate interactions in the adsorption system. Therefore, we plotted the heats of methane adsorption in the as-prepared ZrBDC powder (see Figure 11(a)) and the ZrBDC-PVA pellets (Figure 11(b)) as a function of methane uptake over a wide range of temperatures in order to compare the mechanism of methane adsorption in these Zr-based materials.

As follows from Figures 11(a) and 11(b), at all measured temperatures, the initial differential molar isosteric heats of adsorption are about 19.3-18.9 $\mathrm{kJ} / \mathrm{mol}$. These values are close or exceeded that determined for methane adsorption in most
MOF materials [67, 91], including Zr-based MOFs as Nu$1000(16-18 \mathrm{~kJ} / \mathrm{mol}[92]$ and UiO-type MOFs [78, 93]). The comparison of Figures 11(a) and 11(b) revealed the discrepancy between the functions $q_{\mathrm{st}}=f(a)$ for the $\mathrm{ZrBDC}$ powder and ZrBDC-PVA pellets. The variation in the $q_{\mathrm{st}}$ with an increase in the amount of adsorbed methane molecules reflects the changes in the mechanism of methane adsorption determined by the ratio between $\mathrm{CH}_{4}$-MOF and $\mathrm{CH}_{4}-\mathrm{CH}_{4}$ interactions. The decrease in the value of $q_{\mathrm{st}}$ from the initial value at the early stage of adsorption (up to $a \sim 1 \mathrm{mmol} / \mathrm{g}$ ) to about $12-16 \mathrm{~kJ} / \mathrm{mol}$ (at $a=4-5 \mathrm{mmol} /$ g) observed in the pristine $\mathrm{ZrBDC}$ powder is caused by the gradual occupation of the adsorption sites with different interaction energies by methane molecules, starting the narrowest pores (so-called adsorption pockets with one methane molecule [94]) and $\mathrm{Zr}$ ions [78].

In contrast, the methane adsorption in the ZrBDCPVA pellets is characterized by the extreme function $q_{\mathrm{st}}$ $=f(a)$ with a wide maximum, the amplitude, width and position of which depend on temperature (see Figure 11(b)). Then, the heat of adsorption declines rapidly at higher values of methane uptake. In our opinion, these behaviors can be ascribable to the variations in the porous structure of $\mathrm{ZrBDC}$ caused by the compaction with PVA, namely the distribution and energy level of the adsorption sites. We suppose that the interactions between the binder macromolecules and methane molecules contribute to the heat of adsorption. It should be noted that Stadie et al. observed similar anomalous behaviors for methane adsorption in zeolite-templated carbons and attributed it to the relatively high homogeneity of binding site energies caused by a narrow pore size distribution, periodic spacing, and high content of $\mathrm{sp}^{2}$-hybridized carbon [95]. We agree with the authors of this work that correct estimations of the impacts of intermolecular interactions to the isosteric heat of methane adsorption in ZrBDC-PVA require additional investigations of the adsorption energy of the different adsorption sites.

As follows from Figures 11(a) and 11(b)(curves 2-8) and (curve 7), the drop of $q_{\text {st }}$ slows down within the range of medium values of $3-5$ or $3 \mathrm{mmol} / \mathrm{g}$, which indicates an increase in the contribution from the $\mathrm{CH}_{4}-\mathrm{CH}_{4}$ interactions. The effect of lateral $\mathrm{CH}_{4}-\mathrm{CH}_{4}$ interactions during adsorption in different microporous adsorbents, including $\mathrm{NaX}$ zeolite [96], activated carbons [97, 98], and MOFs [99, 100], was shown experimentally and confirmed by molecular dynamics. The decrease in the heat of adsorption observed at the adsorption values above $6-8 \mathrm{mmol} / \mathrm{g}$ and $253-333 \mathrm{~K}$ can be attributed to a decrease in the intensity of the adsorption process ( $\partial P / \partial a$ increases), accompanied by the enhancement of repulsion due to the reduced distances between methane molecules and pore walls [101]. As the temperature rises, these effects are observed at lower pore loadings due to the increasing contribution from kinetic energy associated with molecular mobility.

At the initial stage of micropore loading: $a<1.2$ $\mathrm{mmol} / \mathrm{g}$ for as-prepared ZrBDC and $a<0.7 \mathrm{mmol} / \mathrm{g}$ for ZrBDC-PVA, the isosteric heat of adsorption is independent of temperature (see Figures 11(a) and 11(b)). 


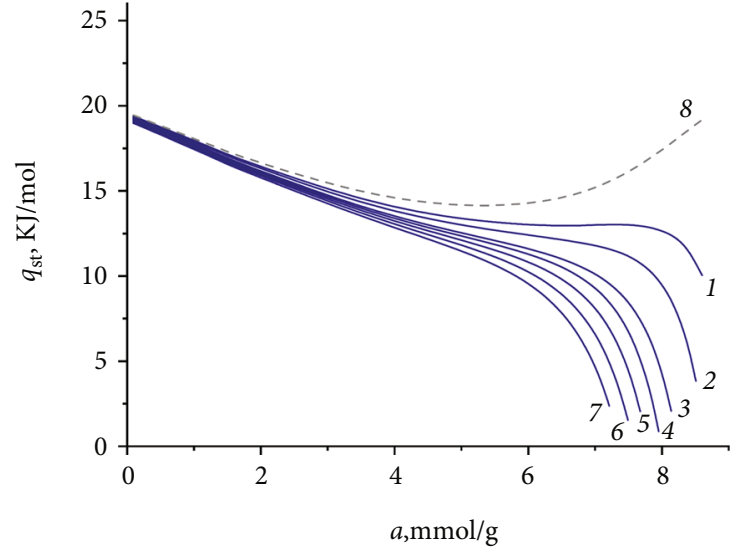

(a)

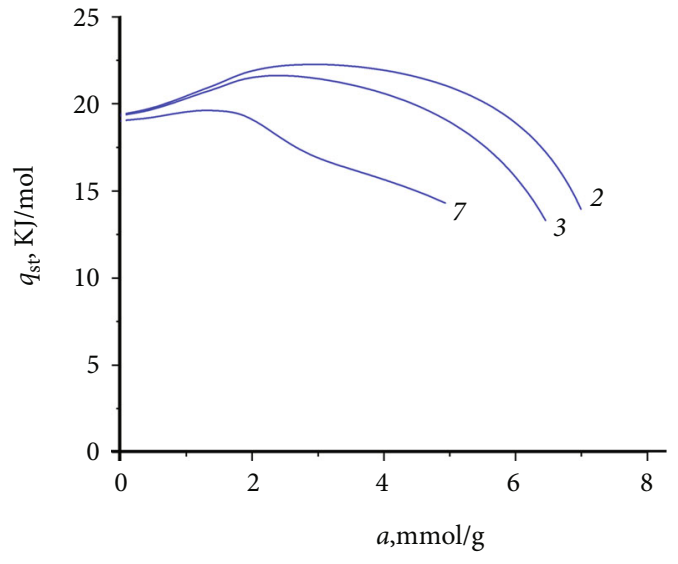

(b)

Figure 11: Differential isosteric heat of methane adsorption onto as-prepared powder ZrBDC (a) and ZrBDC-PVA pellets versus methane adsorption at temperatures K: 253 (1), 273 (2), 293 (3), 303 (4), 313 (5), 323 (6), and 333 (7), calculated using Eq. (7). The dashed line 8 depicts the value of $q_{\text {st }}$ methane adsorption onto as-prepared powder $\operatorname{ZrBDC}$ when $Z=1$ and $(\partial P / \partial a) \approx 0$.

However, with a subsequent increase in methane adsorption, the temperature dependence of the isosteric heat of adsorption is observed for both adsorbents, and it becomes increasingly noticeable as the pore space is filled with methane. The observed divergence in the curves $q_{\mathrm{st}}=f(a$ ) can be interpreted in terms of Eq. (8) by the significant changes in the contributions from the slope of isosteres and the compressibility of the gaseous phase $Z$ with temperature.

These conclusions are confirmed by the temperature dependences of the differential molar isosteric enthalpy of the $\mathrm{ZrBDC}-\mathrm{CH}_{4}$ adsorption system, $h_{1}$, which can be calculated from Eq. (6) using the data of differential isosteric heat of adsorption. Figure 12 shows the temperature dependences of the differential molar enthalpy of the $\mathrm{ZrBDC}-\mathrm{CH}_{4}$ system for different values of methane adsorption. At the initial stage of adsorption, the enthalpy of the adsorption system increases almost linearly with temperature, which is associated with the temperature invariance of the heat of adsorption, $q_{\mathrm{st}} \neq f(T)$, and the linear character of the temperature dependence of the molar enthalpy of an equilibrium gas phase, $h_{\mathrm{g}}$. However, the $h_{1}(T)$ dependences become nonlinear during the methane adsorption: the slope $\partial h_{1}(T) / \partial T$ increases with temperature.

The observed variations of $h_{1}$ during the process of methane adsorption in ZrBDC, which were evaluated from the experimental isotherms of adsorption, are consistent with the computational studies of the enthalpy variations for methane in nanoporous solids. In particular, using the continuous fractional component Monte Carlo algorithm for calculating thermodynamics for the methanezeolite adsorption system, Torres-Knoop et al. showed that the repulsive interactions between an adsorbate molecule and the previously adsorbed species and the adsorbent were responsible for the rearrangements of methane molecules, which lead to the increase in the enthalpy at high micropore loadings close to saturation [101].
The differential molar entropy of the adsorbed methane molecules calculated as

$$
s_{1}=s_{\mathrm{g}}-\frac{q_{\mathrm{st}}}{T}
$$

and represented as a function of methane uptake in Figure 13 reflects the variations in the state of methane molecules during adsorption compared to a gaseous phase.

As can be seen in Figure 13, at the adsorption value of about $1 \mathrm{mmol} / \mathrm{g}$, there is a sharp decrease in entropy, indicating the transfer of methane molecules with three translational, three rotational, and six vibrational degrees of freedom to the bound state on the high-energy adsorption sites. The state of methane molecules adsorbed onto MOF is characterized by reduced mobility, which can be described as probably three rotational and three vibrational degrees of freedom or six vibrational degrees of freedom [99]. In the range of adsorption values of $2-6 \mathrm{mmol} / \mathrm{g}$, the drop in entropy slows down during the gradual methane loading of the pore space and the impact of the $\mathrm{CH}_{4}-\mathrm{CH}_{4}$ interactions. An increase in the $s_{1}(a)$ values is observed when the pore loadings are above $\sim 6 \mathrm{mmol} / \mathrm{g}$, which we attribute to the rearrangement of adsorbed methane molecules close to saturation.

The heat capacity of the $\mathrm{CH}_{4} / \mathrm{ZrBDC}$ adsorption system is essential for the characterization of the system since it determines the thermal stability due to thermal fluctuations during adsorption/desorption cycles. Unlike the heat capacity of any bulk phase, this parameter of the adsorption system depends not only on the pressure and temperature but also on the amount of adsorbed substance. Therefore, a complete thermodynamic description of the adsorption system involves the analysis of the differential molar isosteric heat capacity, $C_{a}$, as a function of all these parameters. The value of $C_{a}$ was calculated using the Kirchhoff equation obtained by differentiating Eq. (11) with respect to 


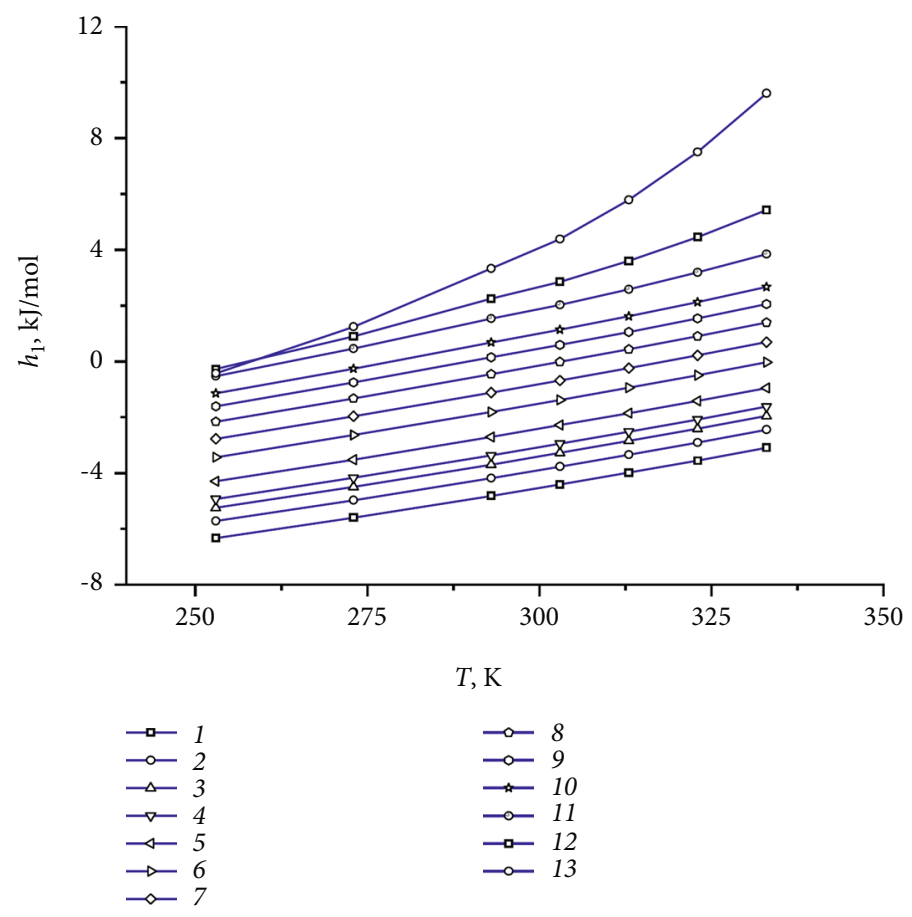

FIGURE 12: Temperature dependence of the differential molar isosteric enthalpy of the ZrBDC-CH4 adsorption system at different values of methane adsorption, mmol/g: 0.1 (1), 0.5 (2), 0.8 (3), 1 (4), 1.4 (5), 2 (6), 2.5 (7), 3 (8), 3.5 (9), 4 (10), 5 (11), 6 (12), and 7 (13). The results of calculations are shown by symbols; the lines are the spline approximation.

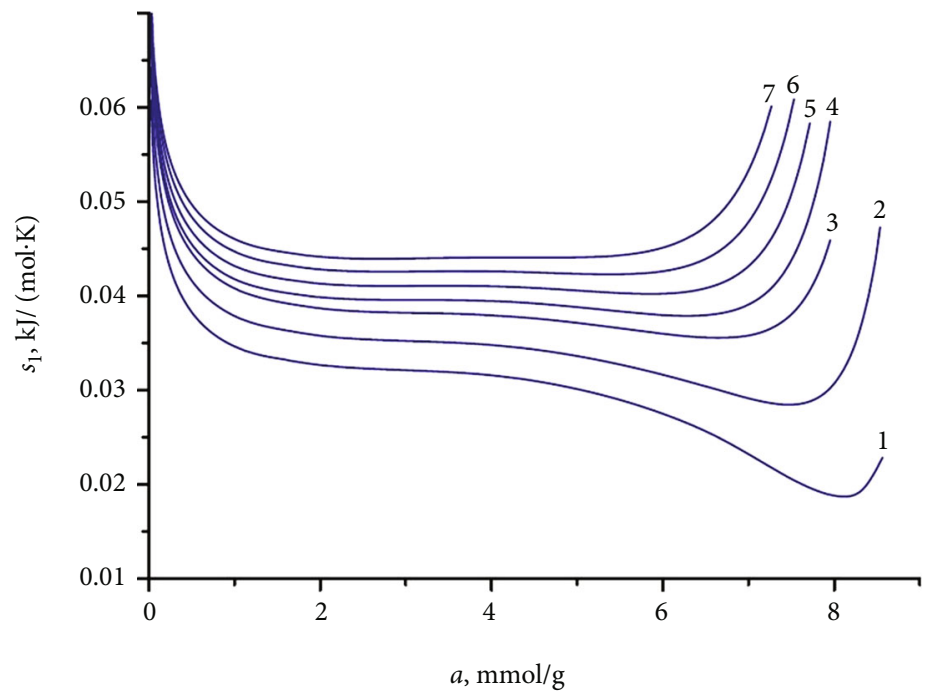

Figure 13: Differential molar isosteric entropy of methane in as-prepared Zr-BDC as a function of the amount of adsorbed methane at temperatures, K: 253 (1), 273 (2), 293 (3), 303 (4), 313 (5), 323 (6), and 333 (7).

temperature [87]:

$$
C_{a}=\left(\frac{\partial h_{1}}{\partial T}\right)_{a}=\left(\frac{\partial h_{g}}{\partial T}\right)_{a}-\left(\frac{\partial q_{s t}}{\partial T}\right)_{a} .
$$

Figure 14 demonstrates the temperature dependence of the differential molar isosteric heat capacity of the ZrBDC$\mathrm{CH}_{4}$ for various amounts of adsorbed methane.
As follows from Figure 14, the differential molar isosteric heat capacity of the $\mathrm{CH}_{4} / \mathrm{ZrBDC}$ adsorption system depends on temperature and increases with adsorption. At low values of methane adsorption, the increase in the isosteric heat capacity of the adsorption system is determined by the temperature dependence of the enthalpy of methane in a gaseous state since, as was shown above: $q_{s t} \neq f(T)$. However, as follows from Figure 11(a), during the process of methane adsorption, the temperature invariance of $q_{\mathrm{st}}$ is replaced by the explicit temperature dependence of $q_{\mathrm{st}}$, which begins to 


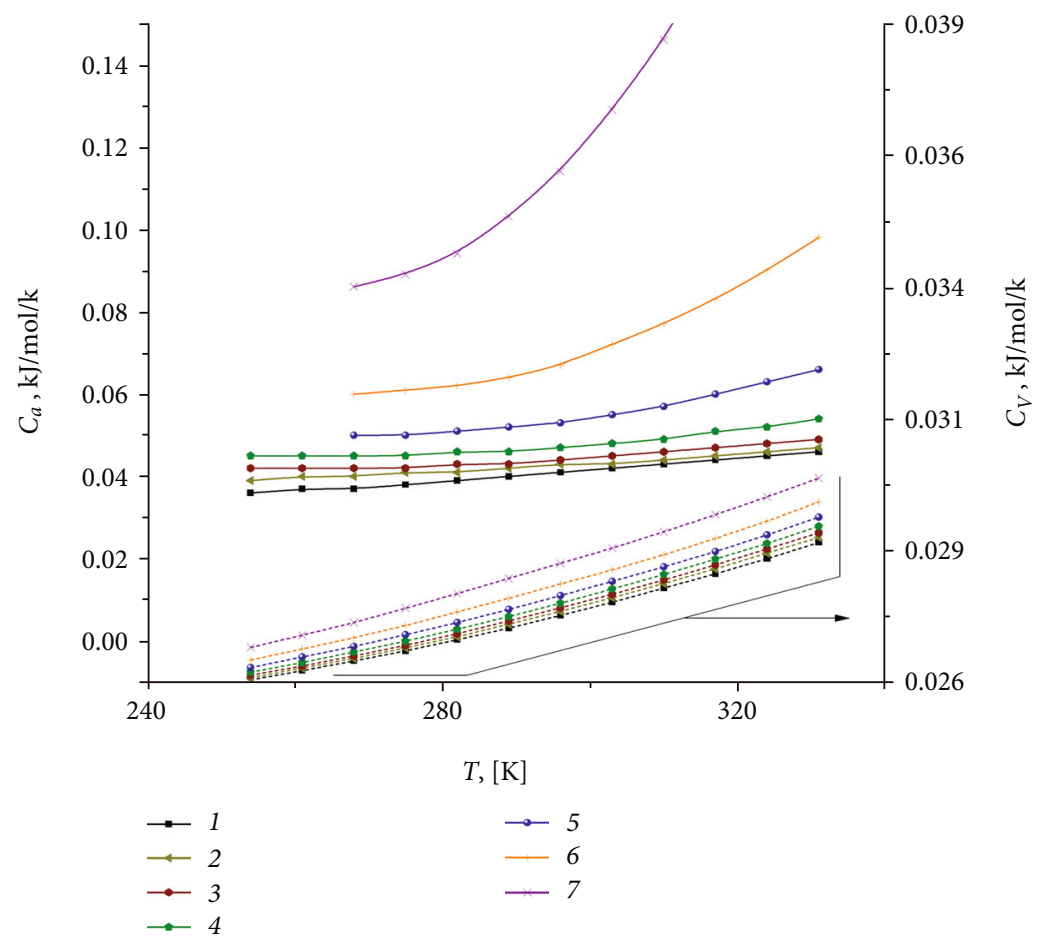

FIgURE 14: Temperature dependences of the differential molar heat capacity of the $\mathrm{CH}_{4} / \mathrm{ZrBDC}$ adsorption system, $C_{a}$, and isochoric heat capacity of the methane gas phase, $C_{v}$, for different amounts of adsorbed methane, mmol/g: 0.1 (1), 2 (2), 3 (4), 4 (5), 5 (6), 6 (7), and 7 (8).

TABLE 3: Thermodynamic characteristics of the model ANG vessel with a mass of $10 \mathrm{~kg}$ loaded with the ZrBDC powder and ZrBDC-PVA pellets at the pressure of $10 \mathrm{MPa}$ and the temperatures of 273,293 , and $333 \mathrm{~K}$.

\begin{tabular}{|c|c|c|c|c|c|}
\hline Sample & Heat capacity, $\mathrm{kJ} / \mathrm{kg} \cdot \mathrm{K}$ & $T, \mathrm{~K}$ & 273 & 293 & 333 \\
\hline \multirow{3}{*}{ ZrBDC powder } & \multirow{3}{*}{8.39} & $a_{\max }, \mathrm{mmol} / \mathrm{g}$ & 8.5 & 7.9 & 6.7 \\
\hline & & $Q, \mathrm{~kJ} / \mathrm{Mol}$ & 118.0 & 108.7 & 92.4 \\
\hline & & $\Delta T, \mathrm{~K}$ & 13.7 & 12.5 & 10.8 \\
\hline \multirow{3}{*}{ ZrBDC-PVA pellets } & \multirow{3}{*}{8.21} & $a_{\max }, \mathrm{mmol} / \mathrm{g}$ & 7.0 & 6.45 & 4.9 \\
\hline & & $Q, \mathrm{~kJ} / \mathrm{Mol}$ & 116.9 & 107.9 & 92.8 \\
\hline & & $\Delta T, \mathrm{~K}$ & 14.1 & 12.6 & 11.1 \\
\hline
\end{tabular}

contribute to the heat capacity of the adsorption system $C_{\mathrm{a}}$ $=f(T)$. The heat capacity of the $\mathrm{CH}_{4} / \mathrm{ZrBDC}$ adsorption system significantly exceeds (by $\sim 2-3$ times) the isochoric heat capacity of the gaseous methane phase, $C_{V}$. This fact indicates a specific highly dispersed state of adsorbed methane in a strong adsorption field generated by a microporous adsorbent [87].

3.7. Thermal Effects in the Model ANG Systems with the $Z r B D C$-Based Adsorbents. In addition to the thermodynamic state functions, the data on differential isosteric heat of methane adsorption onto the ZrBDC-based materials were used to access thermal effects in the model ANG vessels arising during the adsorption (charging) process. For this purpose, we calculate the integral heat of adsorption $Q$ released during adsorption [85]:

$$
Q(T)=\int_{0}^{a} q_{\mathrm{st}}(a)_{T=\mathrm{const}} d a .
$$

Then, the values of $Q$ were used to calculate the variation in temperature $(\Delta T)$ of a model ANG system filled with the ZrBDC powder and the ZrBDC-PVA pellets during adsorption under adiabatic conditions. The heat capacities of the ZrBDC-based materials were evaluated using the empirical Neumann-Koop rule equation [102] and the data in Table 1 . The results of the calculations are listed in Table 3.

According to Table 3, an increase in the temperature of the adsorption process is accompanied by a decrease in the integral heat released in both adsorption systems. A difference between the values of $Q$ observed in the ANG systems with the powdered ZrBDC adsorbent and ZrBDC-PVA pellets reached $1.1 \mathrm{~kJ}$ at $273 \mathrm{~K}$, and it decreased to $0.4 \mathrm{~kJ}$ at $333 \mathrm{~K}$. A slight excess in the heating-up of the ANG system with the pellets can be ascribed to the lower heat capacity of ZrBDC-PVA. Also, the data in Table 3 testified that the heating-up of the ANG system is maximal at low temperatures. Thus, the ZrBDC-PDA pellets demonstrated superiority in the deliverable capacity for methane compared to 
ZrBDC powder (see Figures 10(a) and 10(c)), which was not accompanied by a considerable increase in the temperature of the adsorbent. Indeed, the heating-up of the ANG system loaded with the MOF-based materials occurred much less than that for activated carbons: $\Delta T=53 \mathrm{~K}$, obtained for the values of methane adsorption of $8 \mathrm{mmol} / \mathrm{g}$ at $5 \mathrm{MPa}$ and $273 \mathrm{~K}$ [85]. Thus, the thermal effects arising during the adsorption (charging) process do not threaten the integrity of the MOF-based adsorbent during cyclic operation.

\section{Conclusions}

To conclude, we explored two factors affecting the performance of the ANG system with a MOF-based adsorbent: textural characteristics of the material, including its porous structure and packing density, and thermodynamic behaviors of the entire adsorption system as a whole. These factors affect the deliverable storage capacity and thermal management of the ANG system.

The exploration and comparison of the structural and adsorption properties of a set of Zr-based MOFs: asprepared ZrBDC and three shaped ZrBDC materials, which were prepared by compacting without a binder and mixed with polymer binders such as PVA and CMC, over a range of compaction pressure from 30 to $240 \mathrm{MPa}$ and at $298 \mathrm{~K}$, combined with the data of the tests of the lab-scale ANG tank loaded with the shaped ZrBDC-based pellets brought us to the following conclusions:

(i) The increase in the $\mathrm{ZrBDC}$ material density depends on the compaction pressure and the use of a binder. The highest density of the ZrBDCbased materials was achieved by compacting the as-prepared MOF powder under the pressure of $240 \mathrm{MPa}$ without any binders: $1.6 \mathrm{~g} / \mathrm{cm}^{3}$. The compaction of the ZrBDC powders mixed with the binders PVA or CMC under $240 \mathrm{MPa}$ also led to a more than three-fold increase in their density: up to $1.1 \mathrm{~g} / \mathrm{cm}^{3}$

(ii) Although the binders retain the crystal structure of ZrBDC, they affect the degree of degradation of the porous structure of the shaped $\mathrm{ZrBDC}$ material. The proportion of micro- and mesopores in the shaped ZrBDC-based material occurred to be dependent on the ratio between the molecular size of a binder and pore diameter. PVA was found to be the best binder since it provided a possibility to achieve a minimal loss of micropore volume and specific surface area of the final ZrBDC-based upon compaction

(iii) The compaction of the ZrBDC powder at a minimum pressure of $30 \mathrm{MPa}$ and the use of PVA as a binder, leading to a more than two-fold increase in the packing density and lowest degradation of the porous structure, was sufficient to improve the methane adsorption capacity of the final adsorbent by $\sim 11 \%$ : up to $172 \mathrm{~m}^{3}(\mathrm{NTP}) / \mathrm{m}^{3}$ between 0.1 and $10 \mathrm{MPa}$ at $253 \mathrm{~K}$. This value exceeded that observed for the as-prepared ZrBDC powder and compacted
ZrBDC-F material. Therefore, the ZrBDC-PVA material can be considered as a promising adsorbent for small-scale ANG systems

Thus, the results obtained in this study demonstrated the possibility of such techniques of fabrication of MOF-based adsorbents as compaction for improving their performance for ANG systems. In this regard, it is expected that further progress in fabricating the optimal ZrBDC-based materials which meet the DOE target can be ensured by combining two options: (i) functionalization aimed at an increase in the energy of adsorption and (ii) shaping of these functionalized materials.

The results of measurements of the methane adsorption onto the as-prepared ZrBDC powder and ZrBDC-PVA pellets over the temperature range from 253 to $333 \mathrm{~K}$ served to evaluate the thermodynamic state functions of the adsorption systems, primarily the differential molar isosteric heat of adsorption. The analysis of the thermodynamic functions versus the value of methane uptake within the range of temperatures yielded the following results:

(i) The compaction with PVA altered the behaviors of the differential molar isosteric heat of methane adsorption onto $\mathrm{ZrBDC}$ as a function of methane uptake, which can be ascribed to the changes in the distribution and energy level of adsorption sites in the adsorbent, including narrow pores and access to metal ions

(ii) The variations in the thermodynamic state functions of the $\mathrm{CH}_{4} / \mathrm{ZrBDC}$ adsorption system during adsorption, including the differential molar isosteric heat of adsorption, enthalpy, entropy, and heat capacity, can be attributed to the changes in the ratio of the $\mathrm{CH}_{4}$-adsorbent and $\mathrm{CH}_{4}-\mathrm{CH}_{4}$ interactions, which altered the state of adsorbed molecules from the partially-localized state near the highenergy adsorption sites with restricted mobility to the adsorption molecular clusters, which are rearranged close to saturation

(iii) The temperature changes of ANG systems loaded with the ZrBDC powder and ZrBDC-PVA pellets upon the adsorption (charging) process differ insignificantly. The relatively low values of temperature changes in the ANG tank loaded with the ZrBDCPVA pellets indicate the high operating properties during cyclic work

Evaluating the key thermodynamic quantities is essential for selecting an efficient adsorbent for the ANG systems, novel gas-power drones and vehicles, and corresponding heat-exchange facilities to achieve the maximum storage density and deliverable capacity.

\section{Data Availability}

The experimental data used to support the findings of this study are included within the article. 


\section{Disclosure}

The funders had no role in the design of the study; in the collection, analyses, or interpretation of the data; in the writing of the manuscript; or in the decision to publish the results.

\section{Conflicts of Interest}

The authors declare no conflict of interest.

\section{Acknowledgments}

The experiments were carried out using equipment of the Center of Physical Methods of Investigations of the A.N. Frumkin Institute of Physical Chemistry and Electrochemistry of the Russian Academy of Sciences. SEM and XRD investigation was performed using equipment of Center of Shared Use of IPCE RAS. We thank Dr. A.L. Pulin for his help in measuring low-temperature nitrogen vapor adsorption and Dr. V.V. Vysotskii for his assistance in the SEM experiments and constructive suggestions. The research was carried out within the State Assignment of the Ministry of Science and Higher Education of the Russian Federation No. 122011300053-8.

\section{References}

[1] CO2 Emissions, World Bank, 2015, 2021, http://go.nature .com/yLaqyF.

[2] "Natural Gas by Country," 2021, https:// worldpopulationreview.com/country-rankings/natural-gasby-country.

[3] A. Y. Tsivadze, O. E. Aksyutin, A. G. Ishkov et al., "Metalorganic framework structures: adsorbents for natural gas storage," Russian Chemical Reviews, vol. 88, no. 9, pp. 925978, 2019.

[4] A. Y. Tsivadze, O. E. Aksyutin, A. G. Ishkov et al., "Porous carbon-based adsorption systems for natural gas (methane) storage," Russian Chemical Reviews, vol. 87, no. 10, pp. 950-983, 2018.

[5] T. A. Makal, J.-R. Li, W. Lu, and H.-C. Zhou, "Methane storage in advanced porous materials," Chemical Society Reviews, vol. 41, no. 23, pp. 7761-7779, 2012.

[6] M. I. Khan, T. Yasmin, and A. Shakoor, "Technical overview of compressed natural gas (CNG) as a transportation fuel," Renewable and Sustainable Energy Reviews, vol. 51, pp. 785-797, 2015.

[7] P. Cleaver, M. Johnson, and B. Ho, "A summary of some experimental data on LNG safety," Journal of Hazardous Materials, vol. 140, no. 3, pp. 429-438, 2007.

[8] A. Luketa-Hanlin, "A review of large-scale LNG spills: Experiments and modeling," Journal of Hazardous Materials, vol. 132, no. 2-3, pp. 119-140, 2006.

[9] A. Grint and S. T. Takagishi, "“Adsorbed Natural Gas (ANG)," Research Conducted by Atlanta Gas Light Adsorbent Research Group (AGLARG). Final Report. (19901993)," Gas Research Institute, End-Use Technology Development Division, Des Planes, IL, 1994.

[10] A. A. Fomkin, A. A. Pribylov, A. G. Tkachev et al., "The influence of the structural and energetic characteristics of the microporous structure of carbon adsorbents on hydrogen adsorption," Colloid Journal, vol. 81, no. 5, pp. 607-612, 2019.

[11] I. E. Men'shchikov, A. A. Fomkin, A. Y. Tsivadze, A. V. Shkolin, E. M. Strizhenov, and E. V. Khozina, "Adsorption accumulation of natural gas based on microporous carbon adsorbents of different origin," Adsorption, vol. 23, no. 2-3, pp. 327-339, 2017.

[12] I. Men'shchikov, A. Shiryaev, A. Shkolin, V. Vysotskii, E. Khozina, and A. Fomkin, "Carbon adsorbents for methane storage: genesis, synthesis, porosity, adsorption," Korean Journal of Chemical Engineering, vol. 38, no. 2, pp. 276-291, 2021.

[13] H. Li, K. Wang, Y. Sun, C. T. Lollar, J. Li, and H. C. Zhou, "Recent advances in gas storage and separation using metalorganic frameworks," Materials Today, vol. 21, no. 2, pp. 108-121, 2018.

[14] I. E. Men'shchikov, A. A. Fomkin, A. V. Shkolin, V. Y. Yakovlev, and E. V. Khozina, "Optimization of structural and energy characteristics of adsorbents for methane storage," Russian Chemical Bulletin, vol. 67, no. 10, pp. 1814-1822, 2018.

[15] MOVE Program Overview, "Advanced Research Project Agency, US DOE,” 2012, 2017, http://arpa-e.energy.gov/ s it e s/default/files/documents/files/ MOVEProgramOverview.pdf.

[16] M. Bastos-Neto, D. V. Canabrava, A. E. B. Torres et al., "Effects of textural and surface characteristics of microporous activated carbons on the methane adsorption capacity at high pressures," Applied Surface Science, vol. 253, no. 13, pp. 57215725, 2007.

[17] Y. He, W. Zhou, G. Qian, and B. Chen, "Methane storage in metal-organic frameworks," Chemical Society Reviews, vol. 43, no. 16, pp. 5657-5678, 2014.

[18] A. A. Fomkin, I. E. Men'shchikov, A. A. Pribylov et al., "Methane adsorption on microporous carbon adsorbent with wide pore size distribution," Colloid Journal, vol. 79, no. 1, pp. 144-151, 2017.

[19] K. Thu, Y.-D. Kim, A. B. Ismil, B. B. Saha, and K. C. Ng, "Adsorption characteristics of methane on Maxsorb III by gravimetric method," Applied Thermal Engineering, vol. 72, no. 2, pp. 200-205, 2014.

[20] C. O. Ania and E. Raymundo-Piñero, "Nanoporous carbons with tuned porosity," in Chapter 5 in "Nanoporous Materials for Gas Storage”, K. Kaneko and F. Rodríguez-Reinoso, Eds., pp. 99-135, Springer Nature Pte Ltd, Singapore, 2019.

[21] I. E. Men'shchikov, A. A. Fomkin, Y. A. Romanov et al., "Carbon nanoporous adsorbents prepared from walnut Shell for liquefied natural gas vapor recovery in cryogenic storage systems," Protection of Metals and Physical Chemistry of Surfaces, vol. 56, no. 6, pp. 1122-1133, 2020.

[22] C. E. Wilmer, M. Leaf, C. Y. Lee et al., "Large-scale screening of hypothetical metal-organic frameworks," Nature Chemistry, vol. 4, no. 2, pp. 83-89, 2012.

[23] C. M. Simon, J. Kim, D. A. Gomez-Gualdron et al., "The materials genome in action: identifying the performance limits for methane storage," Energy \& Environmental Science, vol. 10, no. 4, pp. 1190-1199, 2015.

[24] H. M. M. Wen, B. Li, D. Yuan et al., "A porous metal-organic framework with an elongated anthracene derivative exhibiting a high working capacity for the storage of methane," Journal of Materials Chemistry A, vol. 2, no. 29, pp. 11516-11522, 2014. 
[25] B. Li, H.-M. Wen, W. Zhou, J. Q. Xu, and B. Chen, "Porous metal-organic frameworks: promising materials for methane storage," Chem, vol. 1, no. 4, pp. 557-580, 2016.

[26] C. C. Liang, Z. L. Shi, C. T. He et al., "Engineering of Pore Geometry for Ultrahigh Capacity Methane Storage in Mesoporous Metal-Organic Frameworks," Journal of the American Chemical Society, vol. 139, no. 38, pp. 13300-13303, 2017.

[27] A. Ahmed, Y. Liu, J. Purewal et al., "Balancing gravimetric and volumetric hydrogen density in MOFs," Energy \& Environmental Science, vol. 10, no. 11, pp. 2459-2471, 2017.

[28] O. V. Solovtsova, A. V. Shkolin, I. E. Men'shchikov et al., "Functional composite adsorbents based on metal-organic frameworks in a carbon matrix applied for methane storage," Protection of Metals and Physical Chemistry of Surfaces, vol. 55, no. 6, pp. 1080-1084, 2019.

[29] B. P. Prajwal and G. K. Ayappa, "Evaluating methane storage targets: From powder samples to onboard storage systems," Adsorption, vol. 20, no. 5-6, pp. 769-776, 2014.

[30] X.-M. Liu, L.-H. Xie, and Y. Wu, "Recent advances in the shaping of metal-organic frameworks," Inorganic Chemistry Frontiers, vol. 7, no. 15, pp. 2840-2866, 2020.

[31] E. Mahmoud, L. Ali, A. el Sayah et al., "Implementing MetalOrganic Frameworks for Natural Gas Storage," Crystals, vol. 9, no. 8, p. 406, 2019.

[32] B. B. Shah, T. Kundu, and D. Zhao, "Mechanical properties of shaped metal-organic frameworks," Topics in Current Chemistry, vol. 377, no. 5, pp. 25-29, 2019.

[33] V. Stavila, J. Volponi, A. M. Katzenmeyer, M. C. Dixon, and M. D. Allendorf, "Kinetics and mechanism of metal-organic framework thin film growth: systematic investigation of HKUST-1 deposition on QCM electrodes," Chemical Science, vol. 3, no. 5, pp. 1531-1540, 2012.

[34] J. Zhao, M. D. Losego, P. C. Lemaire et al., "Highly adsorptive, MOF-functionalized nonwoven fiber mats for hazardous gas capture enabled by atomic layer deposition," Advanced Materials Interfaces, vol. 1, no. 4, p. 1400040, 2014.

[35] J. Zhao, B. Gong, W. T. Nunn et al., "Conformal and highly adsorptive metal-organic framework thin films via layer-bylayer growth on ALD-coated fiber mats," Journal of Materials Chemistry A, vol. 3, no. 4, pp. 1458-1464, 2015.

[36] C. L. Lin, S. Lirio, Y. T. Chen, C. H. Lin, and H. Y. Huang, "A novel hybrid metal-organic framework-polymeric monolith for solid-phase microextraction," Chemistry - A European Journal, vol. 20, no. 12, pp. 3317-3321.

[37] H. Zhu, X. Yang, E. D. Cranston, and S. Zhu, "Flexible and porous nanocellulose aerogels with high loadings of metalorganic-framework particles for separations applications," Advanced Materials, vol. 28, no. 35, pp. 7652-7657, 2016.

[38] H. Thakkar, S. Eastman, Q. Al-Naddaf, A. A. Rownaghi, and F. Rezaei, "3D-printed metal-organic framework monoliths for gas adsorption processes," ACS Applied Materials \& Interfaces, vol. 9, no. 41, pp. 35908-35916, 2017.

[39] Y. Chen, X. Huang, S. Zhang et al., "Shaping of metal -organic frameworks: from fluid to shaped bodies and robust foams," Journal of the American Chemical Society, vol. 138, no. 34, pp. 10810-10813, 2016.

[40] T. Tian, Z. Zeng, D. Vulpe et al., "A sol-gel monolithic metalorganic framework with enhanced methane uptake," Nature Materials, vol. 17, no. 2, pp. 174-179, 2018.

[41] A. H. Valekar, K.-H. Cho, U. H. Lee et al., "Shaping of porous metal-organic framework granules using mesoporous $\rho$-alu- mina as a binder," RSC Advances, vol. 7, no. 88, pp. 5576755777, 2017.

[42] L. Garzón-Tovar, M. Cano-Sarabia, A. Carné-Sánchez, C. Carbonell, I. Imaz, and D. Maspoch, "A spray-drying continuous-flow method for simultaneous synthesis and shaping of microspherical high nuclearity MOF beads," Reaction Chemistry \& Engineering, vol. 1, no. 5, pp. 533-539, 2016.

[43] D. Crawford, J. Casaban, R. Haydon, N. Giri, T. McNally, and S. L. James, "Synthesis by extrusion: continuous, large-scale preparation of MOFs using little or no solvent," Chemical Science, vol. 6, no. 3, pp. 1645-1649, 2015.

[44] J. Ren, N. M. Musyoka, H. W. Langmi, A. Swartbooi, B. C. North, and M. Mathe, "A more efficient way to shape metal-organic framework (MOF) powder materials for hydrogen storage applications," International Journal of Hydrogen Energy, vol. 40, no. 13, pp. 4617-4622, 2015.

[45] J. Zheng, X. Cui, Q. Yang, Q. Ren, Y. Yang, and H. Xing, "Shaping of ultrahigh-loading MOF pellet with a strongly anti-tearing binder for gas separation and storage," Chemical Engineering Journal, vol. 354, pp. 1075-1082, 2018.

[46] M. Tagliabue, C. Rizzo, R. Millini, P. D. C. Dietzel, R. Blom, and S. Zanardi, "Methane storage on CPO-27-Ni pellets," Journal of Porous Materials, vol. 18, no. 3, pp. 289-296, 2011.

[47] I. Majchrzak-Kucęba and A. Ściubidło, "Shaping metalorganic framework (MOF) powder materials for $\mathrm{CO} 2$ capture applications-a thermogravimetric study," Journal of Thermal Analysis and Calorimetry, vol. 138, no. 6, pp. 41394144, 2019.

[48] M. I. Nandasiri, S. R. Jambovane, B. P. McGrail, H. T. Schaef, and S. K. Nune, "Adsorption, separation, and catalytic properties of densified metal-organic frameworks," Coordination Chemistry Reviews, vol. 311, pp. 38-52, 2016.

[49] J. H. Cavka, S. Jakobsen, U. Olsbye et al., “A New zirconium inorganic building brick forming metal organic frameworks with exceptional stability," Journal of the American Chemical Society, vol. 130, no. 42, pp. 13850-13851, 2008.

[50] Y. Bai, Y. Dou, L. H. Xie, W. Rutledge, J. R. Li, and H. C. Zhou, "Zr-based metal-organic frameworks: design, synthesis, structure, and applications," Chemical Society Reviews, vol. 45, no. 8, pp. 2327-2367, 2016.

[51] J. Ren, H. W. Langmi, B. C. North, M. Mathe, and D. Bessarabov, "Modulated synthesis of zirconium-metal organic framework (Zr-MOF) for hydrogen storage applications," International Journal of Hydrogen Energy, vol. 39, no. 2, pp. 890-895, 2014.

[52] M. Taddei, "When defects turn into virtues: The curious case of zirconium-based metal- organic frameworks," Coordination Chemistry Reviews, vol. 343, pp. 1-24, 2017.

[53] S. E. Bambalaza, H. W. Langmi, R. Mokaya, N. M. Musyoka, J. Ren, and L. E. Khotseng, "Compaction of a zirconium metal-organic framework (UiO-66) for high density hydrogen storage applications," Journal of Materials Chemistry A, vol. 6, no. 46, pp. 23569-23577, 2018.

[54] M. Rubio-Martinez, C. Avci-Camur, A. W. Thornton, I. Imaz, D. Maspoch, and M. R. Hill, "New synthetic routes towards MOF production at scale," Chemical Society Reviews, vol. 46, no. 11, pp. 3453-3480, 2017.

[55] T. H. Bradley, B. A. Moffitt, D. N. Mavris, and D. E. Parekh, "Development and experimental characterization of a fuel cell powered aircraft," Journal of Power Sources, vol. 171, no. 2, pp. 793-801, 2007. 
[56] R. O. Stroman, M. W. Schuette, K. Swider-Lyons, J. A. Rodgers, and D. J. Edwards, "Liquid hydrogen fuel system design and demonstration in a small long endurance air vehicle," International Journal of Hydrogen Energy, vol. 39, no. 21, pp. 11279-11290, 2014.

[57] O. V. Solovtsova, A. L. Pulin, I. E. Men'shchikov et al., "Zrbased metal-organic Nanoporous adsorbents of high density for methane storage," Protection of Metals and Physical Chemistry of Surfaces, vol. 56, no. 6, pp. 1114-1121, 2020.

[58] O. V. Solovtsova, A. V. Shkolin, I. E. Men'shchikov et al., "Functional composite adsorbents of high packing density based on metal-organic framework structures for methane accumulation," Protection of Metals and Physical Chemistry of Surfaces, vol. 55, no. 5, pp. 826-832, 2019.

[59] M. M. Dubinin, "Physical adsorption of gases and vapors in micropores," Progress in Surface and Membrane Science, vol. 9, pp. 1-70, 1975.

[60] M. M. Dubinin, "Fundamentals of the theory of adsorption in micropores of carbon adsorbents: characteristics of their adsorption properties and microporous structures," Carbon, vol. 27, no. 3, pp. 457-467, 1989.

[61] J. P. B. Mota, "Impact of gas composition on natural gas storage by adsorption," AICHE Journal, vol. 45, no. 5, pp. 986996, 1999.

[62] H. Zhang, P. Deria, O. K. Farha, J. T. Hupp, and R. Q. Snurr, "A thermodynamic tank model for studying the effect of higher hydrocarbons on natural gas storage in metalorganic frameworks," Energy \& Environmental Science, vol. 8, no. 5, pp. 1501-1510, 2015.

[63] K. J. Chang and O. Talu, "Behavior and performance of adsorptive natural gas storage cylinders during discharge," Applied Thermal Engineering, vol. 16, no. 5, pp. 359-374, 1996.

[64] O. Talu, "Physical Chemistry and Engineering for Adsorptive Gas Storage in Nanoporous Solids," in Chapter 4 in "Nanoporous Materials for Gas Storage", K. Kaneko and F. Rodríguez-Reinoso, Eds., pp. 65-90, Springer Nature Pte Ltd, Singapore, 2019.

[65] L. Arnold, G. Averlant, S. Marx et al., "Metal organic frameworks for natural gas storage in Vehicles," Chemie-Ingenieur-Technik, vol. 85, no. 11, pp. 1726-1733, 2013.

[66] A. A. Fomkin, "Adsorption of gases, vapors and liquids by microporous adsorbents," Adsorption, vol. 11, no. 3-4, pp. 425-436, 2005.

[67] G. O. Vissers, W. Zhang, O. E. Vilches et al., "Heats of adsorption of $\mathrm{N}_{2}$, CO, Ar, and CH4versus coverage on the Zr-based MOF NU-1000: measurements and DFT calculations," Journal of Physical Chemistry C, vol. 123, no. 11, pp. 6586-6591, 2019.

[68] A. Nuhnen and C. Janiak, "A practical guide to calculate the isosteric heat/enthalpy of adsorption via adsorption isotherms in metal-organic frameworks, MOFs," Dalton Transactions, vol. 49, no. 30, pp. 10295-10307, 2020.

[69] A. V. Shkolin, A. A. Fomkin, I. E. Men'shchikov, E. M. Strizhenov, A. L. Pulin, and E. V. Khozina, "Monolithic microporous carbon adsorbent for low-temperature natural gas storage," Adsorption, vol. 25, no. 8, pp. 1559-1573, 2019.

[70] "GOST 34100.3-2017/ISO/IEC Guide 98-3:2008. Part 3. Uncertainty of measurement. Part 3. Guide to the expression of uncertainty in measurement," 2020, https://files.stroyinf .ru/Data/651/65118.pdf.
[71] E. Lemmon, M. Huber, and M. McLinden, NIST Standard Reference Database 23: Reference Fluid Thermodynamic and Transport Properties-REFPROP, Version 9.1, Natl Std. Ref. Data Series (NIST NSRDS), National Institute of Standards and Technology, Gaithersburg, MD, 2013, https://tsapps .nist.gov/publication/get_pdf.cfm?pub_id=912382.

[72] V. V. Sychev, A. A. Vasserman, V. A. Zagoruchenko, A. D. Kozlov, G. A. Spiridonov, and V. A. Tsymarny, Thermodynamic Properties of Methane, Izd, Standartov, Moscow, 1979, (in Russian).

[73] S. Brunauer, P. H. Emmett, and E. Teller, "Adsorption of gases in multimolecular layers," Journal of the American Chemical Society, vol. 60, no. 2, pp. 309-319, 1938.

[74] S. J. Gregg and K. S. W. Sing, Adsorption, Surface Area and Porosity, Academic Press, London, New York, 1982.

[75] "ISO 697:1981. Surface active agents. Washing powders. Determination of apparent density. Method by measuring the mass of a given volume," 2018, https://www.iso.org/ standard/4897.html.

[76] A. A. Pribylov, V. V. Serpinsky, and S. M. Kalashnikov, "Adsorption of gases by microporous adsorbents under pressures up to hundreds megapascals," Zeolites, vol. 11, no. 8, pp. 846-849, 1991.

[77] O. V. Solovtsova, S. S. Chugaev, I. E. Men'shchikov, A. L. Pulin, A. V. Shkolin, and A. A. Fomkin, "High-density carbon adsorbents for natural gas storage," Colloid Journal, vol. 82, no. 6, pp. 719-726, 2020.

[78] K. Vasanth Kumar, G. Charalambopoulou, M. E. Kainourgiakis, A. Stubos, and T. Steriotis, "Insights on the physical adsorption of hydrogen and methane in UiO series of MOFs using molecular simulations," Computational \& Theoretical Chemistry, vol. 1061, pp. 36-45, 2015.

[79] S. Vandenbrande, T. Verstraelen, J. J. Gutiérrez-Sevillano, M. Waroquier, and V. van Speybroeck, "Methane adsorption in Zr-based MOFs: comparison and critical evaluation of force fields," Physical Chemistry C, vol. 121, no. 45, pp. 25309-25322.

[80] K. H. Meyer and L. Misch, "Positions des atomes dans le nouveau modèle spatial de la cellulose," Helvetica Chimica Acta, vol. 20, no. 1, pp. 232-244, 1937.

[81] B. P. Nikolsky, Ed., Handbook for chemist (Spravochnik khimika), Leningrad, 1971.

[82] V. A. Bakaev, "The statistical thermodynamics of adsorption equilibriums in the case of zeolites," Doklady Akademii Nauk SSSR, vol. 167, pp. 369-372, 1966.

[83] A. A. Pribylov and K. A. Murdmaa, "Gas Adsorption onto an MN-270 polymer adsorbent within a Supercritical Range of temperatures and pressures," Protection of Metals and Physical Chemistry of Surfaces, vol. 56, no. 2, pp. 245251, 2020.

[84] I. Men'shchikov, A. V. Shkolin, E. M. Strizhenov et al., “Thermodynamic behaviors of adsorbed methane storage systems based on nanoporous carbon adsorbents prepared from coconut shells," Nanomaterials, vol. 10, no. 11, p. 2243, 2020.

[85] I. Men'shchikov, A. V. Shkolin, E. V. Khozina, and A. Fomkin, "Thermodynamics of adsorbed methane storage systems based on peat-derived activated carbons," Nanomaterials, vol. 10, no. 7, pp. 1379-1398, 2020.

[86] "ISO 868:2003. Plastics and ebonite - Determination of indentation hardness by means of a durometer (Shore hardness)," 2003, https://www.iso.org/ru/standard/34804.html. 
[87] T. L. Hill, Theory of physical adsorption in advances in catalysis and related subjects, vol. 4, Acad. Press, New York, NY, USA, 1952.

[88] V. A. Bakaev, Molecular Theory of Physical Adsorption, [Ph.D. thesis], Moscow State University, Moscow, Russia, 1990.

[89] L. G. Shekhovtsova and A. A. Fomkin, "Discrete site model for methane adsorption on microporous adsorbents," Bulletin of the Academy of Sciences of the USSR Division of Chemical Science, vol. 39, no. 5, pp. 867-870, 1990.

[90] H. Wu, T. Yildirim, and W. Zhou, "Exceptional mechanical stability of highly porous zirconium metal-organic framework UiO-66 and its important implications," Journal of Physical Chemistry Letters, vol. 4, no. 6, pp. 925-930, 2013.

[91] K. Konstas, T. Osl, Y. Yang et al., "Methane storage in metal organic frameworks," Journal of Materials Chemistry, vol. 22, no. 33, pp. 16698-16708, 2012.

[92] S. K. Bhatia and A. L. Myers, "Optimum conditions for adsorptive storage," Langmuir, vol. 22, no. 4, pp. 16881700, 2006.

[93] S. Øien-Ødegaard, B. Bouchevreau, K. Hylland et al., "UiO67-type metal-organic frameworks with enhanced water stability and methane adsorption capacity," Inorganic Chemistry, vol. 55, no. 5, pp. 1986-1991, 2016.

[94] D. Gómez-Gualdrón, C. M. Simon, W. Lassman et al., "Impact of the strength and spatial distribution of adsorption sites on methane deliverable capacity in nanoporous materials," Chemical Engineering Science, vol. 159, pp. 18-30, 2017.

[95] N. P. Stadie, M. Murialdo, C. C. Ahn, and B. Fultz, “Anomalous Isosteric enthalpy of adsorption of methane on zeolitetemplated carbon," Journal of the American Chemical Society, vol. 135, no. 3, pp. 990-993, 2013.

[96] E. V. Chkhaidze, A. A. Fomkin, V. V. Serpinskii, and G. V. Tsitsishvili, "Thermodynamics of the adsorption of methane on NaX zeolite," Russian Chemical Bulletin, vol. 35, no. 2, pp. 252-255, 1986.

[97] A. V. Shkolin, A. A. Fomkin, A. Y. Tsivadze, K. M. Anuchin, I. E. Men'shchikov, and A. L. Pulin, "Experimental study and numerical modeling: methane adsorption in microporous carbon adsorbent over the subcritical and supercritical temperature regions," Protection of Metals and Physical Chemistry of Surfaces, vol. 52, no. 6, pp. 955-963, 2016.

[98] K. M. Anuchin, A. A. Fomkin, A. P. Korotych, and A. M. Tolmachev, "Adsorption concentration of methane. Dependence of adsorbate density on the width of slit-shaped micropores in activated carbons," Protection of Metals and Physical Chemistry of Surfaces, vol. 50, no. 2, pp. 173-177, 2014.

[99] K. Sillar and J. Sauer, "Ab initio prediction of adsorption isotherms for small molecules in metal-organic frameworks: the effect of lateral interactions for methane/CPO-27-mg," Journal of the American Chemical Society, vol. 134, no. 44, pp. 18354-18365, 2012.
[100] Z. Hulvey, B. Vlaisavljevich, J. A. Mason et al., "Critical Factors Driving the High Volumetric Uptake of Methane in $\mathrm{Cu}_{3}(\mathrm{btc})_{2}$," Journal of the American Chemical Society, vol. 137, no. 33, pp. 10816-10825, 2015.

[101] A. Torres-Knoop, A. Poursaeidesfahani, T. J. H. Vlugt, and D. Dubbeldam, "Behavior of the enthalpy of adsorption in nanoporous materials close to saturation conditions," Journal of Chemical Theory and Computation, vol. 13, no. 7, pp. 3326-3339, 2017.

[102] J. E. Hurst Jr. and B. Keith Harrison, "Estimation of liquid and solid heat capacities using a modified Kopp's rule," Chemical Engineering Communications, vol. 112, no. 1, pp. 21-30, 1992. 